\title{
Convergence Order in Trajectory Estimation by Piecewise-Cubics and Exponential Parameterization
}

\author{
Ryszard Kozera ${ }^{a, b, c}$ and Magdalena Wilkołazka ${ }^{b}$ \\ ${ }^{a}$ Faculty of Applied Informatics and Mathematics, Warsaw University of Life \\ Sciences - SGGW \\ Nowoursynowska str. 159, 02-776 Warsaw, Poland \\ ${ }^{b}$ Faculty of Mathematics, Informatics and Landscape Architecture, The John \\ Paul II Catholic University of Lublin \\ Konstantynów 1H, 20-708 Lublin, Poland \\ ${ }^{c}$ Department of Computer Science and Software Engineering, The University \\ of Western Australia \\ 35 Stirling Highway, WA 6009 Crawley, Perth, Australia \\ E-mail(corresp.): ryszard.kozera@gmail.com \\ E-mail: magda.wilkolazka@gmail.com
}

Received February 9, 2018; revised November 18, 2018; accepted November 18, 2018

\begin{abstract}
This paper discusses the problem of estimating the trajectory of the unknown curve $\gamma$ from the sequence of $m+1$ interpolation points $Q_{m}=\left\{\gamma\left(t_{i}\right)\right\}_{i=0}^{m}$ in arbitrary Euclidean space $\mathbb{E}^{n}$. The respective knots $\mathcal{T}_{m}=\left\{t_{i}\right\}_{i=0}^{m}$ (in ascending order) are assumed to be unknown. Such $Q_{m}$ is coined reduced data. In our setting, a piecewise-cubic Lagrange interpolation $\hat{\gamma}_{3}:[0, \hat{T}] \rightarrow \mathbb{E}^{n}$ is applied to fit $Q_{m}$. Here, the missing knots $\mathcal{T}_{m}$ are replaced by their estimates $\hat{\mathcal{T}}_{m}=\left\{\hat{t}_{i}\right\}_{i=0}^{m}$ in accordance with the exponential parameterization. The latter is controlled by a single parameter $\lambda \in[0,1]$. This work analyzes the intrinsic asymptotics in approximating $\gamma$ by $\hat{\gamma}_{3}$ based on the exponential parameterization and $Q_{m}$. The multiple goals are achieved. Firstly, the existing result established for $\lambda=1$ (i.e. for the cumulative chord parameterization) is extended to the remaining cases of $\lambda \in[0,1)$ and more-or-less uniformly sampled $Q_{m}$. As demonstrated herein, a quartic convergence order $\alpha(1)=4$ in trajectory estimation drops discontinuously to the linear one $\alpha(\lambda)=1$, for all $\lambda \in[0,1)$. Secondly, the asymptotics derived in this paper is also analytically proved to be sharp with the aid of illustrative examples. Thirdly, the latter is verified in affirmative upon conducting numerical testing. Next, the necessity of more-or-less uniformity imposed on $Q_{m}$ is shown to be indispensable. In addition, several sufficient conditions for $\hat{\gamma}_{3}$ to be
\end{abstract}

Copyright (C) 2019 The Author(s). Published by VGTU Press

This is an Open Access article distributed under the terms of the Creative Commons Attribution License (http://creativecommons.org/licenses/by/4.0/), which permits unrestricted use, distribution, and reproduction in any medium, provided the original author and source are credited. 
reparameterizable to the domain of $\gamma$ are formulated. Lastly, the motivation for using the exponential parameterization with $\lambda \in[0,1)$ is also outlined.

Keywords: interpolation, reduced data, convergence order and sharpness.

AMS Subject Classification: 65D05; 65D10; 65D18.

\section{Introduction}

Let $\gamma:[0, T] \rightarrow \mathbb{E}^{n}$ be a smooth regular curve (i.e. $\dot{\gamma}(t) \neq 0$ ) over $t \in[0, T]$, for $0<T<\infty$. The term reduced data $Q_{m}$ determines $m+1$ interpolation points $\left\{q_{i}\right\}_{i=0}^{m}$ (with $q_{i}=\gamma\left(t_{i}\right)$ ) in arbitrary Euclidean space $\mathbb{E}^{n}$. The corresponding interpolation knots $\mathcal{T}=\left\{t_{i}\right\}_{i=0}^{m}$ satisfying $t_{0}=0<t_{1}<\ldots<t_{m}=T$ are assumed here to be unknown. Any fitting scheme $\hat{\gamma}$ interpolating $Q_{m}$ with some pre-selected knots' estimates $\hat{\mathcal{T}}=\left\{\hat{t}_{i}\right\}_{i=0}^{m} \approx \mathcal{T}$ subject to $q_{i}=\hat{\gamma}\left(\hat{t}_{i}\right)$ is called non-parametric interpolation (see e.g. [3], [14] or [15]).

Having chosen an interpolation scheme $\hat{\gamma}:[0, \hat{T}] \rightarrow \mathbb{E}^{n}$ and some substitutes $\left\{\hat{t}_{i}\right\}_{i=0}^{m}$ of the respective missing knots $\left\{t_{i}\right\}_{i=0}^{m}$, the question of the intrinsic asymptotic order $\alpha$ in $\gamma$ approximation by $\hat{\gamma}$ arises naturally. Recall first the basic definition (see e.g. [1], [6] or [19]):

Definition 1. We say that $\left\{t_{i}\right\}_{i=0}^{m}$ is admissible if:

$$
\lim _{m \rightarrow \infty} \delta_{m} \rightarrow 0^{+}, \text {where } \delta_{m}=\max _{1 \leq i \leq m}\left\{t_{i}-t_{i-1}: i=1,2, \ldots, m\right\} .
$$

The following subclass of (1.1) is here of particular relevance (see [9]):

Definition 2. The sampling $\left\{t_{i}\right\}_{i=0}^{m}$ is more-or-less uniform if for some constants $0<K_{\mathrm{l}} \leq K_{\mathrm{u}}$ and sufficiently large $m: \frac{K_{l}}{m} \leq t_{i}-t_{i-1} \leq \frac{K_{u}}{m}$ holds for all $i=1,2, \ldots, m$. Equivalently, the last two inequalities can be replaced by

$$
\beta \delta_{m} \leq t_{i+1}-t_{i} \leq \delta_{m}
$$

satisfied for some $\beta \in(0,1]$ and sufficiently large $m$.

Recall now the following (see e.g. [1] or [6]):

Definition 3. Consider a family $\left\{f_{\delta_{m}}, \delta_{m}>0\right\}$ of functions $f_{\delta_{m}}:[0, T] \rightarrow \mathbb{E}$. We say that $f_{\delta_{m}}$ is of order $O\left(\delta_{m}^{\alpha}\right)$ (denoted as $f_{\delta_{m}}=O\left(\delta_{m}^{\alpha}\right)$ ), if there is a constant $K>0$ such that, for some $\bar{\delta}>0$ the inequality $\left|f_{\delta_{m}}(t)\right| \leq K \delta_{m}^{\alpha}$ holds for all $\delta_{m} \in(0, \bar{\delta})$, uniformly over $[0, T]$. In case of vector-valued functions $F_{\delta_{m}}:[0, T] \rightarrow \mathbb{E}^{n}$ by $F_{\delta_{m}}=O\left(\delta_{m}^{\alpha}\right)$ it is understood that $\left\|F_{\delta_{m}}\right\|=O\left(\delta_{m}^{\alpha}\right)$.

This paper deals with a special family of discrete exponential parametetrizations designed to estimate the missing knots $\left\{t_{i}\right\}_{i=0}^{m}$ (see e.g. [14]) according to:

$$
\hat{t}_{0, \lambda}=0, \quad t_{i+1, \lambda}=\hat{t}_{i, \lambda}+\left\|q_{i+1}-q_{i}\right\|^{\lambda}
$$

for arbitrary $\lambda \in[0,1]$ and $i=0,1,2, \ldots, m-1$. In order to preserve the ascending order of $\hat{\mathcal{T}}_{\lambda}=\left\{\hat{t}_{i, \lambda}\right\}_{i=0}^{m}$ an extra constraint $q_{i+1} \neq q_{i}$ is imposed here on the admitted class of reduced data $Q_{m}$. Visibly, the case of $\lambda=0$ 
in (1.3) yields the uniform distribution of $\left\{t_{i, 0}\right\}_{i=0}^{m}$ with no account given to the geometrical layout of $Q_{m}$. On the other hand, the so-called cumulative chord parameterization (i.e. when $\lambda=1$ in (1.3)) incorporates the dispersion of $Q_{m}$. Noticeably, the case of $\lambda=0,5$ in (1.3) covers the so-called centripetal parameterization (see [14]). To abbreviate the notation, from now on (unless needed otherwise) the parameter $\lambda$ in subscript of $\hat{t}_{i, \lambda}$ determined by (1.3) is omitted.

Upon selecting specific interpolation scheme (e.g. piecewise $r$-degree Lagrange polynomials $\hat{\gamma}=\hat{\gamma}_{r}$, with either $r=2$ or $r=3$ ) and then varying the parameter $\lambda \in[0,1]$ in (1.3) different interpolants $\hat{\gamma}_{r}$ can be constructed. Recall that for $\left\{q_{i}, q_{i+1}, q_{i+2}\right\}$ with $r=2$ the Lagrange quadratic $\hat{\gamma}_{2, i}:\left[\hat{t}_{i}, \hat{t}_{i+2}\right] \rightarrow \mathbb{E}^{n}$ satisfies $\hat{\gamma}_{2, i}\left(\hat{t}_{i+j}\right)=q_{i+j}$, for $j=0,1,2$ (where e.g. $\hat{t}_{i+j}$ are defined according to (1.3)). The track sum of $\hat{\gamma}_{2, i}$ defines a piecewise-quadratic interpolant $\hat{\gamma}_{2}:\left[\hat{t}_{0}=0, \hat{t}_{m}\right] \rightarrow \mathbb{E}^{n}$ based on reduced data $Q_{m}$ and $\left\{\hat{t}_{i}\right\}_{i=0}^{m}$. Upon extending the domain of each $\hat{\gamma}_{2, i}$ from $\hat{I}_{i}=\left[\hat{t}_{i}, \hat{t}_{i+2}\right]$ into $\mathbb{R}$ one defines a modified collection of quadratics $\check{\gamma}_{2, i}: \mathbb{R} \rightarrow \mathbb{E}^{n}$ fulfilling the interpolation conditions $\check{\gamma}_{2, i}\left(\hat{t}_{i+j}\right)=q_{i+j}$ and satisfying $\left.\check{\gamma}_{2, i}\right|_{\left[\hat{t}_{i}, \hat{t}_{i+2}\right]}=\hat{\gamma}_{2, i}$. The latter yields $\check{\gamma}_{2, i} \circ \psi_{i}=\hat{\gamma}_{2, i} \circ \psi_{i}$, for arbitrary mapping $\psi_{i}: I_{i}=\left[t_{i}, t_{i+2}\right] \rightarrow\left[\hat{t}_{i}, \hat{t}_{i+2}\right]$. In particular, this holds for $\psi_{i}$ reparameterizing $I_{i}$ into $\hat{I}_{i}$ (i.e. for $\psi_{i}$ strictly increasing). However, for $\psi_{i}\left(\left[t_{i}, t_{i+2}\right]\right) \nsubseteq\left[\hat{t}_{i}, \hat{t}_{i+2}\right]$ the asymptotic analysis of the difference $\hat{\gamma}_{2, i} \circ \psi_{i}-\gamma$ over $I_{i}$ needs to be adjusted to $\check{\gamma}_{2, i} \circ \psi_{i}-\gamma$ over $I_{i}$.

Assume now that $\psi_{i}=\psi_{2, i}$ is a unique Lagrange quadratic satisfying $\psi_{2, i}\left(t_{i+j}\right)=\hat{t}_{i+j}$ (for $\left.j=0,1,2\right)$, where $\hat{t}_{i+j}$ are defined as in (1.3). The following result holds (see [9] and [17]):

Theorem 1. Let $\gamma$ be a $C^{3}([0, T])$ regular curve in $\mathbb{E}^{n}$ sampled more-or-less uniformly (see Definition 2). Assume that $Q_{m}$ forms reduced data with the unknown knots estimated by (1.3). Then, uniformly over $[0, T]$ :

$$
\check{\gamma}_{2, i} \circ \psi_{2, i}-\gamma=O\left(\delta_{m}^{1}\right), \quad \text { for } \lambda \in[0,1)
$$

and for either uniform $\left\{t_{i}\right\}_{i=0}^{m}=i T / m$ or $\lambda=1$ we have:

$$
\check{\gamma}_{2, i} \circ \psi_{2, i}-\gamma=O\left(\delta_{m}^{3}\right) \text {. }
$$

Moreover, if the mapping $\psi_{2, i}$ is a reparameterization of $I_{i}$ into $\hat{I}_{i}$ (e.g. when $\left\{t_{i}\right\}_{i=0}^{m}$ is uniform or for $\lambda=1$ ) then, in both (1.4) and (1.5), a phrase $\check{\gamma}_{2, i}$ can be replaced by $\hat{\gamma}_{2, i}$.

Note that if $\lambda=1$ the condition (1.2) can be replaced by the weaker assumption imposed on samplings $\mathcal{T}$ i.e. by the condition (1.1). The asymptotics from Theorem 1 is also proved to be sharp (see [9]) understood as:

DEFINITION 4. For a given interpolation scheme $\hat{\gamma}$ based on reduced data $Q_{m}$ and some estimates $\hat{\mathcal{T}}$ of the unknown knots $\mathcal{T}$ (and subject to some selected mapping $\psi:[0, T] \rightarrow[0, \hat{T}])$ we say that asymptotics $\gamma-\hat{\gamma} \circ \psi=O\left(\delta_{m}^{\alpha}\right)$ over $[0, T]$ is sharp within the prescribed family of curves $\gamma \in \mathcal{J}$ and family of samplings $\mathcal{T} \in \mathcal{K}$, if for some $\gamma \in \mathcal{J}$ and some sampling $\mathcal{T} \in \mathcal{K}$, there exist $\bar{t} \in[0, T]$ and some positive constant $K$ such that $\|(\hat{\gamma} \circ \psi)(\bar{t})-\gamma(\bar{t})\|=$ $K \delta_{m}^{\alpha}+O\left(\delta_{m}^{\eta}\right)$, where $\eta>\alpha$. 
In particular, for sharpness of (1.4): $\mathcal{J}=C^{3}$, the more-or-less uniform samplings (1.2) forms $\mathcal{K}, \hat{\gamma}=\check{\gamma}_{2}$ (built from $\hat{\gamma}_{2}$ ) with $\psi=\psi_{2}$. Here $\hat{\gamma}_{2}$ (and $\psi_{2}$ ) is the track-sum of $\hat{\gamma}_{2, i}$ (and of $\psi_{2, i}$ ).

Visibly, (1.4) and (1.5) introduce the unexpected left-hand side discontinuity in convergence orders $\alpha(\lambda)$ at $\lambda=1$ dropping sharply from $\alpha(1)=3$ to $\alpha(\lambda)=1$ (for all $\lambda \in\left[0,1\right.$ ). The sufficient conditions guaranteeing $\psi_{2, i}$ : $\left[t_{i}, t_{i+2}\right] \rightarrow\left[\hat{t}_{i}, \hat{t}_{i+2}\right]$ to be a genuine reparameterization for some $\lambda \in[0,1)$ and $\beta \in(0,1]$ are given in [9] or [10].

On the other hand, a full convergence analysis for piecewise cubic Lagrange interpolation $\hat{\gamma}_{3, i}:\left[\hat{t}_{i}, \hat{t}_{i+3}\right] \rightarrow \mathbb{E}^{n}$ based on exponential parameterization (1.3) and $\psi_{3, i}: J_{i}=\left[t_{i}, t_{i+3}\right] \rightarrow \hat{J}_{i}=\left[\hat{t}_{i}, \hat{t}_{i+3}\right]$ (satisfying $\psi_{3, i}\left(t_{i+j}\right)=\hat{t}_{i+j}$, for $j=0,1,2,3)$ is so-far completed merely for $\lambda=1$. Indeed, as shown in [13] and [17] a sharp quartic convergence order in estimating $\hat{\gamma}_{3, i} \circ \psi_{3, i}-\gamma$ over $[0, T]$ prevails. Here, similarly to the case of $r=2$ and $\lambda=1$ the cubic $\psi_{3, i}$ defines a genuine reparameterization.

\section{Main results and motivation}

We emphasize now the main contributions and the motivation of this paper:

1. This paper extends the analysis of approximation error in $\hat{\gamma}_{3, i} \circ \psi_{3, i}-\gamma$ for the remaining $\lambda \in[0,1)$ - the case $\lambda=1$ is covered in [17]. In fact, as proved in the next section an analogous sharp deceleration in convergence orders claimed by Theorem 1 appears also for $r=3$ and $\lambda \in[0,1]$. Before formulating the main result we adopt a similar notation for the adjusted cubics $\check{\gamma}_{3, i}: \mathbb{R} \rightarrow \mathbb{E}^{n}$ satisfying $\check{\gamma}_{3, i}\left(\hat{t}_{i+j}\right)=q_{i+j}$ and $\left.\check{\gamma}_{3, i}\right|_{\left[\hat{t}_{i}, \hat{t}_{i+3}\right]}=\hat{\gamma}_{3, i}$. As previously if $\psi_{3, i}$ maps $J_{i}$ into $\hat{J}_{i}$ then $\check{\gamma}_{3, i} \circ \psi_{3, i}=\hat{\gamma}_{3, i} \circ \psi_{3, i}$. The latter holds e.g. if $\psi_{3, i}$ defines a genuine reparameterization of $J_{i}$ into $\hat{J}_{i}$ (i.e. $\dot{\psi}_{3, i}>0$ ).

The following result is proved in this paper (see Section 3):

Theorem 2. Let $\gamma$ be a $C^{4}([0, T])$ regular curve in $\mathbb{E}^{n}$ sampled more-or-less uniformly (see Definition 2). Suppose that $Q_{m}$ defines reduced data with the missing knots $\mathcal{T}=\left\{t_{i}\right\}_{i=0}^{m}$ compensated according to (1.3). Then the following holds (uniformly over $[0, T]$ ):

$$
\check{\gamma}_{3, i} \circ \psi_{3, i}-\gamma=O\left(\delta_{m}^{1}\right), \quad \text { for } \lambda \in[0,1) .
$$

In addition, for either $\mathcal{T}_{u}=\left\{\frac{i T}{m}\right\}_{i=0}^{m}$ or $\lambda=1$ (for $\lambda=1$ - see [17]) we obtain:

$$
\check{\gamma}_{3, i} \circ \psi_{3, i}-\gamma=O\left(\delta_{m}^{4}\right) .
$$

Moreover, if additionally the mapping $\psi_{3, i}$ is asymptotically a reparameterization of $J_{i}$ into $\hat{J}_{i}$ (e.g. when $\left\{t_{i}\right\}_{i=0}^{m}$ is uniform or for $\lambda=1$ ) then, in both (2.1) and (2.2), the curve $\check{\gamma}_{3, i}$ can be replaced by $\hat{\gamma}_{3, i}$.

Recently a similar linear convergence order as in (2.1) is established in [7] for the so-called modified Hermite interpolant $\hat{\gamma}_{H} \in C^{1}$ combined with the exponential parameterization (1.3) and $\lambda \in[0,1$ ) (the case of $\lambda=1$ was covered in [6] or [8]). The proof used in [7] exploits the final claim of Theorem 2. 
Indeed, in order to substantiate $\rho=\hat{\gamma}_{H} \circ \phi-\gamma=O\left(\delta_{m}^{1}\right)$ one decomposes $\rho$ into $\rho=\left(\hat{\gamma}_{H} \circ \phi-\hat{\gamma}_{3} \circ \psi_{3}\right)+\left(\hat{\gamma}_{3} \circ \psi_{3}-\gamma\right)$. The $O\left(\delta_{m}^{1}\right)$ asymptotics for the second component in $\rho$ follows directly from (2.1). The linear asymptotics determining the first component $\hat{\gamma}_{H} \circ \phi-\hat{\gamma}_{3} \circ \psi_{3}$ results upon conducting more advanced argument (as compared to Section 3) - see [7].

2. The convergence orders established in Theorem 2 are justified analytically as sharp in accordance with Definition 4 - see Section 5.

3. Section 7 supplements a) and b) with the numerical tests conducted in Mathematica confirming experimentally the sharpness of the asymptotics determined in (2.1) and (2.2).

4. In Section 6 (see Example 3) it is also demonstrated with the aid of symbolic computation performed in Mathematica that more-or-less uniformity (1.2) cannot be dropped in Theorem 2. Remarkably, by [6] or [17] the case of $\lambda=1$ does not require more-or-less uniformity (1.2). Here, merely the general class of admissible samplings (1.1) is sufficient to ascertain (1.5) or (2.2). Noticeably, for the uniform sampling $\mathcal{T}_{u}$ the coefficient $\beta=1$ as introduced in (1.2).

5. Sufficient conditions enforcing $\psi_{3, i}$ to be a genuine reparameterization of $J_{i}$ into $\hat{J}_{i}$ are additionally formulated in the closing part of Section 4 (they are visualized in Figure 1). In general, the question of $\psi:[0, T] \rightarrow[0, \hat{T}]$ rendering a reparameterization is vital e.g. for estimating the length $d(\gamma)=\int_{0}^{T}\|\dot{\gamma}(t)\| d t$ of $\gamma$ with $d(\hat{\gamma})=\int_{0}^{\hat{T}}\left\|\hat{\gamma}^{\prime}(\hat{t})\right\| d \hat{t}$ (see e.g. [2] and [6]). However, in certain circumstances, the non-parameterization cases (yielding loops in trajectory path $\hat{\gamma} \circ \psi$ over some segments $J_{i}$ ) may also be desirable for some specific applications like robot or airborne flying devise trajectory planning (e.g. for inspecting electrical poles by drones).

6. Fitting reduced data $Q_{m}$ (dense or sparse) constitutes an important task in computer graphics and vision, engineering, physics and in other applications like medical or biological image processing - see e.g. [4], [5], [11], [14] or [18]. The resulting abrupt deceleration in estimating $\hat{\gamma}_{3} \circ \psi_{3}-\gamma=O\left(\delta_{m}^{\alpha(\lambda)}\right)$ dropping from $\alpha(1)=4$ to $\alpha(\lambda)=1$ (with $\lambda \in[0,1)$ ) yields an interesting theoretical result complementing [17], Theorem 1 and [7]. However, the interpolation conditions $\hat{\gamma}\left(\hat{t}_{i}\right)=q_{i}$ are frequently accompanied with some extra collateral constraints (e.g. minimizing the curvature of the $\hat{\gamma}$ in curve modelling - see [12]). The exponential parameterization (1.3) may serve here as a possible tool in selecting the respective "optimal knots" $\hat{\mathcal{T}}_{\lambda}$. Indeed, upon relaxing a single parameter $\lambda \in[0,1)$ at the cost of maintaining a slow order $\alpha(\lambda)=1$ in $\gamma$ estimation, an optimal $\lambda_{o p t} \in[0,1)$ can be found to reinforce the extra constraint in question. Evidently, such degree of freedom is lost once $\lambda$ in (1.3) is fixed. In particular, the latter eventuates for $\lambda=1$ frozen. Here despite resulting a fast convergence order in trajectory estimation (as in (1.5) or in (2.2)) no flexibility of adjusting optimal parameter $\lambda$ enforcing additional constraint is left anymore. 


\section{The proof of Theorem 2}

In this section the proof of Theorem 2 is given. In doing so recall first the Hadamard's Lemma (see e.g. [16]):

Lemma 1. Let $f:[a, b] \rightarrow \mathbb{E}^{n}$ be of class $C^{l}$, where $l \geq 1$ and assume that $f\left(t_{0}\right)=\mathbf{0}$, for some $t_{0} \in(a, b)$. There exists a $C^{l-1}$ function $g:[a, b] \rightarrow \mathbb{E}^{n}$ for which $f(t)=\left(t-t_{0}\right) g(t)$. Additionally, $g(t)=O\left(\frac{d f}{d t}\right)$.

If function $f(t)$ has multiple zeros $t_{0} \leq t_{1} \leq \ldots \leq t_{k}$ with $k+1 \leq l$, then Hadamard's Lemma applied $k+1$ times yields:

$$
f(t)=\left(t-t_{0}\right)\left(t-t_{1}\right) \ldots\left(t-t_{k}\right) h(t),
$$

where $h$ is $C^{l-(k+1)}$ and $h=O\left(d^{k+1} f / d t^{k+1}\right)$. From now on, to justify Theorem 2 , the abbreviated notation for both cubics $\psi_{3, i}=\psi_{i}$ and $\check{\gamma}_{3, i}=\check{\gamma}_{i}$ is used.

Proof. The proof of Theorem 2 admits an arbitrary $\lambda \in[0,1)$ and any more-or-less uniform sampling. In contrast, the special case $\lambda=1$ (omitted here as already justified in [17]) extends to all admissible samplings (1.1) but is still restricted to $\gamma \in C^{4}$. Noticeably, for $\lambda=1$ each cubic $\psi_{i}$ defines a genuine reparameterization of $J_{i}$ into $\hat{J}_{i}$ - see [6] or [17].

Define now the following "error function" $f_{i}(t)=\left(\check{\gamma}_{i} \circ \psi_{i}\right)(t)-\gamma(t)$. Combining $f_{i}\left(t_{i+j}\right)=0$ (for $j=0,1,2,3$ ) with Hadamard's Lemma yields (see here $(3.1))$ :

$$
\begin{aligned}
f_{i}(t) & =\left(t-t_{i}\right)\left(t-t_{i+1}\right)\left(t-t_{i+2}\right)\left(t-t_{i+3}\right)\left[\left(\check{\gamma}_{i} \circ \psi_{i}\right)^{(4)}(t)-\gamma^{(4)}(t)\right] \\
& =O\left(\delta_{m}^{4}\right) \cdot O\left(\left(\check{\gamma}_{i} \circ \psi_{i}\right)^{(4)}(t)-\gamma^{(4)}(t)\right) .
\end{aligned}
$$

Since $\check{\gamma}_{i}$ and $\psi_{i}$ are cubics the chain rule applied to (3.2) results in:

$$
\left(\check{\gamma}_{i} \circ \psi_{i}\right)^{(4)}(t)=6 \check{\gamma}_{i}^{\prime \prime \prime}(\hat{t}) \dot{\psi}_{i}^{2}(t) \ddot{\psi}_{i}(t)+3 \check{\gamma}_{i}^{\prime \prime}(\hat{t}) \ddot{\psi}_{i}^{2}(t)+4 \check{\gamma}_{i}^{\prime \prime}(\hat{t}) \dot{\psi}_{i}(t) \dddot{\psi}_{i}(t)
$$

with the respective derivatives over $t$ or $\hat{t}=\psi_{i}(t)$ expressed by either dotted or apostrophed notations, respectively. Coupling the latter with (3.2) and $\gamma \in C^{4}$ defined over a compact set $[0, T]$ leads to:

$$
\begin{aligned}
f_{i}(t)= & O\left(\delta_{m}^{4}\right)\left[O\left(\check{\gamma}_{i}^{\prime \prime \prime}(\hat{t}) \dot{\psi}_{i}^{2}(t) \ddot{\psi}_{i}(t)\right)\right. \\
& \left.+O\left(\check{\gamma}_{i}^{\prime \prime}(\hat{t}) \ddot{\psi}_{i}^{2}(t)\right)+O\left(\check{\gamma}_{i}^{\prime \prime}(\hat{t}) \dot{\psi}_{i}(t) \dddot{\psi}_{i}(t)\right)+O(1)\right] .
\end{aligned}
$$

We pass now to the determination of the respective asymptotics for all contributing terms appearing in (3.4).

\subsection{Estimation of derivatives of $\psi_{i}$}

To examine the asymptotics of the derivatives of $\psi_{i}$, recall now Newton's interpolation formula (see [1] or [19]) reading as:

$$
\begin{aligned}
\psi_{i}(t)= & \psi_{i}\left[t_{i}\right]+\psi_{i}\left[t_{i}, t_{i+1}\right]\left(t-t_{i}\right)+\psi_{i}\left[t_{i}, t_{i+1}, t_{i+2}\right]\left(t-t_{i}\right)\left(t_{i}-t_{i+1}\right) \\
& +\psi_{i}\left[t_{i}, t_{i+1}, t_{i+2}, t_{i+3}\right]\left(t-t_{i}\right)\left(t-t_{i+1}\right)\left(t-t_{i+2}\right)
\end{aligned}
$$


over $J_{i}$. Hence

$$
\begin{aligned}
& \dot{\psi}_{i}(t)=\psi_{i}\left[t_{i}, t_{i+1}\right]+\left(2 t-t_{i}-t_{i+1}\right) \psi_{i}\left[t_{i}, t_{i+1}, t_{i+2}\right]+\left[\left(t-t_{i+1}\right)\left(t-t_{i+2}\right)\right. \\
& \left.\quad+\left(t-t_{i}\right)\left(t-t_{i+1}\right)+\left(t-t_{i}\right)\left(t-t_{i+2}\right)\right] \psi_{i}\left[t_{i}, t_{i+1}, t_{i+2}, t_{i+3}\right] .
\end{aligned}
$$

Consequently

$$
\begin{aligned}
& \ddot{\psi}_{i}(t)=2 \psi_{i}\left[t_{i}, t_{i+1}, t_{i+2}\right]+2\left(3 t-t_{i+2}-t_{i+1}-t_{i}\right) \psi_{i}\left[t_{i}, t_{i+1}, t_{i+2}, t_{i+3}\right], \\
& \dddot{\psi}_{i}(t)=6 \psi_{i}\left[t_{i}, t_{i+1}, t_{i+2}, t_{i+3}\right] .
\end{aligned}
$$

Thus by (3.5) and (3.6), the examination of the asymptotics of $\dot{\psi}_{i}, \ddot{\psi}_{i}, \dddot{\psi}_{i}$ over each $J_{i}$ relies on estimating the asymptotic orders of divided differences $\psi_{i}\left[t_{i}, t_{i+1}\right], \psi_{i}\left[t_{i}, t_{i+1}, t_{i+2}\right]$ and $\psi_{i}\left[t_{i}, t_{i+1}, t_{i+2}, t_{i+3}\right]$. Taylor's expansion combined with (1.3) (see also [9], where quadratics $\psi_{2, i}$ instead of cubics $\psi_{3, i}$ are analyzed) yields:

$$
\begin{aligned}
\psi_{i}\left[t_{i}, t_{i+1}\right] & =\frac{\psi_{i}\left(t_{i+1}\right)-\psi_{i}\left(t_{i}\right)}{t_{i+1}-t_{i}}=\frac{\left.\| \gamma\left(t_{i+1}\right)-\gamma\left(t_{i}\right)\right) \|^{\lambda}}{t_{i+1}-t_{i}} \\
& =\left(t_{i+1}-t_{i}\right)^{\lambda-1}+O\left(\left(t_{i+1}-t_{i}\right)^{\lambda+1}\right)=O\left(\delta_{m}^{\lambda-1}\right) .
\end{aligned}
$$

Note that since $\lambda-1<0$ the last step in (3.7) resorts to more-or-less uniformity since then $0<\left(t_{i+1}-t_{i}\right)^{\lambda-1} \leq \beta^{\lambda-1} \delta_{m}^{1-\lambda}$. In addition, the justification of (3.7) relies also on the regularity of the curve $\gamma$. Indeed, such curves are reparameterizable to arc-length rendering $\|\dot{\gamma}(t)\|=1$ (for each $t \in[0, T]$ ) see [15] and [2]. Thus, the dot product $\langle\dot{\gamma}(t) \mid \ddot{\gamma}(t)\rangle \equiv 0$ nullifies slow terms in Taylor expansion of $\left\|\gamma\left(t_{i+1}\right)-\gamma\left(t_{i}\right)\right\|$. Similarly, for $j=0,1$ we have

$$
\begin{aligned}
& \psi_{i}\left[t_{i+j+1}, t_{i+j+2}\right]=\left(t_{i+j+2}-t_{i+j+1}\right)^{\lambda-1} \\
& +O\left(\left(t_{i+j+2}-t_{i+j+1}\right)^{\lambda+1}\right)=O\left(\delta_{m}^{\lambda-1}\right) .
\end{aligned}
$$

The formula for $\psi_{i}\left[t_{i}, t_{i+1}, t_{i+2}\right]$ and $0<\left(t_{i+j+1}-t_{i+j}\right)\left(t_{i+2}-t_{i}\right)^{-1}<1$ (for $j=0,1)$ coupled together with (3.7) and (3.8) yields:

$$
\begin{aligned}
\psi_{i}\left[t_{i}, t_{i+1}, t_{i+2}\right]= & \frac{\left(t_{i+2}-t_{i+1}\right)^{\lambda-1}-\left(t_{i+1}-t_{i}\right)^{\lambda-1}}{t_{i+2}-t_{i}} \\
& +O\left(\left(t_{i+2}-t_{i+1}\right)^{\lambda}\right)+O\left(\left(t_{i+1}-t_{i}\right)^{\lambda}\right)=O\left(\delta_{m}^{\lambda-2}\right) .
\end{aligned}
$$

Again (1.2) is exploited while justifying the last step of (3.9). Analogously:

$$
\begin{aligned}
\psi_{i}\left[t_{i+1}, t_{i+2}, t_{i+3}\right]= & \frac{\left(t_{i+3}-t_{i+2}\right)^{\lambda-1}-\left(t_{i+2}-t_{i+1}\right)^{\lambda-1}}{t_{i+3}-t_{i+1}} \\
& +O\left(\left(t_{i+3}-t_{i+2}\right)^{\lambda}\right)+O\left(\left(t_{i+2}-t_{i+1}\right)^{\lambda}\right)=O\left(\delta_{m}^{\lambda-2}\right) .
\end{aligned}
$$

Thus by (3.9) and (3.10) the fourth divided difference

$$
\begin{gathered}
\psi_{i}\left[t_{i}, t_{i+1}, t_{i+2}, t_{i+3}\right]=\frac{\left(t_{i+3}-t_{i+2}\right)^{\lambda-1}-\left(t_{i+2}-t_{i+1}\right)^{\lambda-1}}{\left(t_{i+3}-t_{i+1}\right)\left(t_{i+3}-t_{i}\right)} \\
-\frac{\left(t_{i+2}-t_{i+1}\right)^{\lambda-1}-\left(t_{i+1}-t_{i}\right)^{\lambda-1}}{\left(t_{i+2}-t_{i}\right)\left(t_{i+3}-t_{i}\right)}+\frac{O\left(\left(t_{i+3}-t_{i+2}\right)^{\lambda+1}\right)+O\left(\left(t_{i+2}-t_{i+1}\right)^{\lambda+1}\right)}{\left(t_{i+3}-t_{i+1}\right)\left(t_{i+3}-t_{i}\right)} \\
\quad+\frac{O\left(\left(t_{i+2}-t_{i+1}\right)^{\lambda+1}\right)+O\left(\left(t_{i+1}-t_{i}\right)^{\lambda+1}\right)}{\left(t_{i+2}-t_{i}\right)\left(t_{i+3}-t_{i}\right)}
\end{gathered}
$$


Upon observing $0<\left(t_{i+j+2}-t_{i+j+1}\right)^{2}\left(\left(t_{i+3}-t_{i+1}\right)\left(t_{i+3}-t_{i}\right)\right)^{-1}<1$ and $0<\left(t_{i+j+1}-t_{i+j}\right)^{2}\left(\left(t_{i+2}-t_{i}\right)\left(t_{i+3}-t_{i}\right)\right)^{-1}<1$ (for $j=0,1$ ) formula (3.11) used together with more-or-less uniformity leads to:

$$
\psi_{i}\left[t_{i}, t_{i+1}, t_{i+2}, t_{i+3}\right]=O\left(\delta_{m}^{\lambda-3}\right)+O\left(\delta_{m}^{\lambda-1}\right)=O\left(\delta_{m}^{\lambda-3}\right) .
$$

Coupling together (3.5), (3.7), (3.9) and (3.12) leads to:

$$
\dot{\psi}_{i}(t)=O\left(\delta_{m}^{\lambda-1}\right)+O\left(\delta_{m}\right) \cdot O\left(\delta_{m}^{\lambda-2}\right)+O\left(\delta_{m}^{2}\right) \cdot O\left(\delta_{m}^{\lambda-3}\right)=O\left(\delta_{m}^{\lambda-1}\right)
$$

Similarly by (3.6), (3.7), (3.9) and (3.12) the following holds:

$$
\ddot{\psi}_{i}(t)=O\left(\delta_{m}^{\lambda-2}\right)+O\left(\delta_{m}\right) \cdot O\left(\delta_{m}^{\lambda-3}\right)=O\left(\delta_{m}^{\lambda-2}\right), \quad \dddot{\psi}_{i}(t)=O\left(\delta_{3}^{\lambda-3}\right) .
$$

The latter exploits the inequalities

$$
\left|t-t_{k}\right| \leq t_{i+3}-t_{i}=\left(t_{i+1}-t_{i}\right)+\left(t_{i+2}-t_{i+1}\right)+\left(t_{i+3}-t_{i+2}\right) \leq 3 \delta_{m},
$$

holding for each $t, t_{k} \in\left[t_{i}, t_{i+3}\right]$ (with $k=0,1,2$ ).

For the special case of uniform samplings $\mathcal{T}_{u}=\left\{\frac{i T}{m}\right\}_{i=0}^{m}$ (where $\beta=1$ see $(1.2))$ by (3.7), (3.9) and (3.11) we have (for $\lambda \in[0,1)$ :

$$
\begin{aligned}
\psi_{i}\left[t_{i}, t_{i+1}\right] & =\delta_{m}^{\lambda-1}+O\left(\delta_{m}^{\lambda+1}\right), \quad \psi_{i}\left[t_{i}, t_{i+1}, t_{i+2}\right]=O\left(\delta_{m}^{\lambda}\right), \\
\psi_{i}\left[t_{i}, t_{i+1}, t_{i+2}, t_{i+3}\right] & =O\left(\delta_{m}^{\lambda-1}\right) .
\end{aligned}
$$

Consequently for $\beta=1$, by (3.5), (3.6) and (3.15) we have (over each $J_{i}$ ):

$$
\dot{\psi}_{i}(t)=\delta_{m}^{\lambda-1}+O\left(\delta_{m}^{\lambda+1}\right), \quad \ddot{\psi}_{i}(t)=O\left(\delta_{m}^{\lambda}\right) \quad \text { and } \quad \dddot{\psi}_{i}(t)=O\left(\delta_{m}^{\lambda-1}\right) .
$$

Hence by (3.16), $\dot{\psi}_{i}>0$ asymptotically. Thus for uniform samplings $\{i T / m\}_{i=0}^{m}$ $\psi_{i}$ defines a genuine reparameterization of $J_{i}$ into $\hat{J}_{i}$, for sufficiently large $m$.

\subsection{Estimation of derivatives of $\check{\gamma}_{i}$}

We estimate now the derivatives of $\check{\gamma}_{i}:\left[t_{i}, t_{i+3}\right] \rightarrow \mathbb{E}^{n}$ present in $(3.2)$ and (3.3). Again Newton's interpolation formula (see e.g. [1]) applied to $\check{\gamma}_{i}$ yields (for any $\hat{t} \in \mathbb{R})$ :

$$
\begin{aligned}
\check{\gamma}_{i}(\hat{t})= & \check{\gamma}_{i}\left[\hat{t}_{i}\right]+\check{\gamma}_{i}\left[\hat{t}_{i}, \hat{t}_{i+1}\right]\left(\hat{t}-\hat{t}_{i}\right)+\check{\gamma}_{i}\left[\hat{t}_{i}, \hat{t}_{i+1}, \hat{t}_{i+2}\right]\left(\hat{t}-\hat{t}_{i}\right)\left(\hat{t}_{i}-\hat{t}_{i+1}\right) \\
& +\check{\gamma}_{i}\left[\hat{t}_{i}, \hat{t}_{i+1}, \hat{t}_{i+2}, \hat{t}_{i+3}\right]\left(\hat{t}-\hat{t}_{i}\right)\left(\hat{t}-\hat{t}_{i+1}\right)\left(\hat{t}-\hat{t}_{i+2}\right) .
\end{aligned}
$$

Hence

$$
\begin{aligned}
\check{\gamma}_{i}^{\prime \prime}(\hat{t}) & =2 \hat{\gamma}_{i}\left[\hat{t}_{i}, \hat{t}_{i+1}, \hat{t}_{i+2}\right]+2\left(3 \hat{t}-\hat{t}_{i+2}-\hat{t}_{i+1}-t_{i}\right) \check{\gamma}_{i}\left[\hat{t}_{i}, \hat{t}_{i+1}, \hat{t}_{i+2}, \hat{t}_{i+3}\right] \\
\check{\gamma}_{i}^{\prime \prime \prime}(\hat{t}) & =6 \check{\gamma}_{i}\left[\hat{t}_{i}, \hat{t}_{i+1}, \hat{t}_{i+2}, \hat{t}_{i+3}\right] .
\end{aligned}
$$

As previously, the orders of $\check{\gamma}_{i}\left[\hat{t}_{i}, \hat{t}_{i+1}\right], \check{\gamma}_{i}\left[\hat{t}_{i}, \hat{t}_{i+1}, \hat{t}_{i+2}\right]$ and $\check{\gamma}_{i}\left[\hat{t}_{i}, \hat{t}_{i+1}, \hat{t}_{i+2}, \hat{t}_{i+3}\right]$ need to be examined. In doing so, observe first that:

$$
\check{\gamma}_{i}\left[\hat{t}_{i}, \hat{t}_{i+1}\right]=\frac{\check{\gamma}_{i}\left(\hat{t}_{i+1}\right)-\check{\gamma}_{i}\left(\hat{t}_{i}\right)}{\hat{t}_{i+1}-\hat{t}_{i}}=\frac{\gamma\left(t_{i+1}\right)-\gamma\left(t_{i}\right)}{\left\|\gamma\left(t_{i+1}\right)-\gamma\left(t_{i}\right)\right\|^{\lambda}} .
$$


Taylor's expansion applied to $\gamma$ yields:

$$
\gamma\left(t_{i+1}\right)-\gamma\left(t_{i}\right)=\left(t_{i+1}-t_{i}\right)\left[\dot{\gamma}\left(t_{i}\right)+\frac{\left(t_{i+1}-t_{i}\right)}{2} \ddot{\gamma}\left(t_{i}\right)+O\left(\left(t_{i+1}-t_{i}\right)^{2}\right)\right] .
$$

Since $\|\dot{\gamma}(t)\|=1$ (and thus $\langle\dot{\gamma}(t) \mid \ddot{\gamma}(t)\rangle=0$ ) we arrive at:

$$
\begin{aligned}
\hat{t}_{i+1}-\hat{t}_{i} & \left.=\| \gamma\left(t_{i+1}\right)-\gamma\left(t_{i}\right)\right) \|^{\lambda}=\left(t_{i+1}-t_{i}\right)^{\lambda}\left[1+O\left(\left(t_{i+1}-t_{i}\right)^{2}\right)\right]^{\frac{\lambda}{2}} \\
& =\left(t_{i+1}-t_{i}\right)^{\lambda}\left[1+O\left(\left(t_{i+1}-t_{i}\right)^{2}\right)\right] .
\end{aligned}
$$

The last step exploits $(1+x)^{\frac{\lambda}{2}}=1+\frac{\lambda}{2} x+O\left(x^{2}\right)$ (for $x$ separated from -1$)$. Hence by (3.18), (3.19) and (3.20) we obtain

$$
\begin{gathered}
\check{\gamma}_{i}\left[\hat{t}_{i}, \hat{t}_{i+1}\right]=\frac{\left(t_{i+1}-t_{i}\right)^{1-\lambda}\left[\dot{\gamma}\left(t_{i}\right)+\frac{\left(t_{i+1}-t_{i}\right)}{2} \ddot{\gamma}\left(t_{i}\right)+O\left(\left(t_{i+1}-t_{i}\right)^{2}\right)\right]}{1+O\left(\left(t_{i+1}-t_{i}\right)^{2}\right)} \\
=\left(t_{i+1}-t_{i}\right)^{1-\lambda}\left[\dot{\gamma}\left(t_{i}\right)+\frac{\left(t_{i+1}-t_{i}\right)}{2} \ddot{\gamma}\left(t_{i}\right)+O\left(\left(t_{i+1}-t_{i}\right)^{2}\right)\right]
\end{gathered}
$$

as $(1+O(x))^{-1}=1+O(x)$ (for $x$ separated from -1$)$. Analogously

$$
\begin{aligned}
& \check{\gamma}_{i}\left[\hat{t}_{i+1}, \hat{t}_{i+2}\right]=\left(t_{i+2}-t_{i+1}\right)^{1-\lambda}\left[\dot{\gamma}\left(t_{i+1}\right)\right. \\
& \left.\quad+\frac{\left(t_{i+2}-t_{i+1}\right)}{2} \ddot{\gamma}\left(t_{i+1}\right)+O\left(\left(t_{i+2}-t_{i+1}\right)^{2}\right)\right] .
\end{aligned}
$$

By $(3.21),(3.22)$ and $\hat{t}_{i+k+2}-\hat{t}_{i+k}=\left(\hat{t}_{i+k+2}-\hat{t}_{i+k+1}\right)+\left(\hat{t}_{i+k+1}-\hat{t}_{i+k}\right)$ (for $k=0,1)$ the asymptotic bound for $\check{\gamma}_{i}\left[\hat{t}_{i+k}, \hat{t}_{i+k+1}, \hat{t}_{i+k+2}\right]=a$

$$
\begin{aligned}
a= & \frac{\left(t_{i+k+2}-t_{i+k+1}\right)^{1-\lambda}\left[\dot{\gamma}\left(t_{i+k+1}\right)+\frac{t_{i+k+2}-t_{i+k+1}}{2} \ddot{\gamma}\left(t_{i+k+1}\right)\right]}{\left(\hat{t}_{i+k+2}-\hat{t}_{i+k+1}\right)+\left(\hat{t}_{i+k+1}-\hat{t}_{i+k}\right)} \\
& -\frac{\left(t_{i+k+1}-t_{i+k}\right)^{1-\lambda}\left[\dot{\gamma}\left(t_{i+k}\right)+\frac{t_{i+k+1}-t_{i+k}}{2} \ddot{\gamma}\left(t_{i+k}\right)\right]}{\left(\hat{t}_{i+k+2}-\hat{t}_{i+k+1}\right)+\left(\hat{t}_{i+k+1}-\hat{t}_{i+k}\right)} \\
& +\frac{O\left(\left(t_{i+k+2}-t_{i+k+1}\right)^{2}\right)+O\left(\left(t_{i+k+1}-t_{i+k}\right)^{2}\right)}{\left(\hat{t}_{i+k+2}-\hat{t}_{i+k+1}\right)+\left(\hat{t}_{i+k+1}-\hat{t}_{i+k}\right)}
\end{aligned}
$$

Using hybrids of $(3.20)$ with $(3.23)$ renders $\left\|\check{\gamma}_{i}\left[\hat{t}_{i+k}, \hat{t}_{i+k+1}, \hat{t}_{i+k+2}\right]\right\|=b$

$$
\begin{aligned}
b \leq & \frac{\left(t_{i+k+2}-t_{i+k+1}\right)^{1-2 \lambda}\left\|\dot{\gamma}\left(t_{i+k+1}\right)+\frac{t_{i+k+2}-t_{i+k+1}}{2} \ddot{\gamma}\left(t_{i+k+1}\right)\right\|}{1+O\left(\left(t_{i+k+2}-t_{i+k+1}\right)^{2}\right)} \\
& +\frac{\left(t_{i+k+1}-t_{i+k}\right)^{1-2 \lambda}\left\|\dot{\gamma}\left(t_{i+k}\right)+\frac{t_{i+k+1}-t_{i+k}}{2} \ddot{\gamma}\left(t_{i+k}\right)\right\|}{1+O\left(\left(t_{i+k+1}-t_{i+k}\right)^{2}\right)} \\
& +\frac{\left\|O\left(\left(t_{i+k+2}-t_{i+k+1}\right)^{2-\lambda}\right)\right\|}{1+O\left(\left(t_{i+k+2}-t_{i+k+1}\right)^{2}\right)}+\frac{\left\|O\left(\left(t_{i+k+1}-t_{i+k}\right)^{2-\lambda}\right)\right\|}{1+O\left(\left(t_{i+k+1}-t_{i+k}\right)^{2}\right)}
\end{aligned}
$$

In the latter the asymptotic positivity of both factors from (3.20) is used as for the exponential parameterization (1.3) with $q_{i+1} \neq q_{i}$ we have $\hat{t}_{l}-\hat{t}_{s}>0$ for $l>s$. Since $1-2 \lambda<2-\lambda($ for $\lambda \in[0,1))$ and $f(x)=(1+x)^{-1}=1+O(x)$ 
(holding for $x$ separated from -1 ), more-or-less uniformity of $\left\{t_{i}\right\}_{i=0}^{m}$ (needed when $1-2 \lambda<0)$ combined with $\|\dot{\gamma}(t)\|^{2}=\langle\dot{\gamma}(t) \mid \dot{\gamma}(t)\rangle \equiv 1$ leads to:

$$
\left\|\check{\gamma}_{i}\left[\hat{t}_{i+k}, \hat{t}_{i+k+1}, \hat{t}_{i+k+2}\right]\right\|=O\left(\delta_{m}^{1-2 \lambda}\right)+O\left(\delta_{m}^{2-\lambda}\right)=O\left(\delta_{m}^{1-2 \lambda}\right),
$$

which by Definition 3 reformulates into:

$$
\check{\gamma}_{i}\left[\hat{t}_{i+k}, \hat{t}_{i+k+1}, \hat{t}_{i+k+2}\right]=O\left(\delta_{m}^{1-2 \lambda}\right) .
$$

In order to examine the asymptotics of $\check{\gamma}_{i}\left[\hat{t}_{i}, \hat{t}_{i+1}, \hat{t}_{i+2}, \hat{t}_{i+3}\right]$, formula (3.23) and $0<\hat{t}_{i+3}-\hat{t}_{i}=\left(\hat{t}_{i+3}-\hat{t}_{i+2}\right)+\left(\hat{t}_{i+2}-\hat{t}_{i+1}\right)+\left(\hat{t}_{i+1}-\hat{t}_{i}\right)$ are combined together. Thus as previously for exponential parameterization (1.3) we have $\left(\hat{t}_{i+3}-\hat{t}_{i}\right)^{-1} \leq\left(\hat{t}_{i+l+1}-\hat{t}_{i+l}\right)^{-1}$ (for $\left.l=0,1,2\right)$. Hence by $(3.23)$ the fourth divided difference

$$
\begin{aligned}
& \check{\gamma}_{i}\left[\hat{t}_{i}, \hat{t}_{i+1}, \hat{t}_{i+2}, \hat{t}_{i+3}\right]=\frac{\check{\gamma}_{i}\left[\hat{t}_{i+1}, \hat{t}_{i+2}, \hat{t}_{i+3}\right]-\check{\gamma}_{i}\left[\hat{t}_{i}, \hat{t}_{i+1}, \hat{t}_{i+2}\right]}{\hat{t}_{i+3}-\hat{t}_{i}} \\
& =\frac{1}{\hat{t}_{i+3}-\hat{t}_{i}}\left(\frac{\left(t_{i+3}-t_{i+2}\right)^{1-\lambda}\left[\dot{\gamma}\left(t_{i+2}\right)+\frac{t_{i+3}-t_{i+2}}{2} \ddot{\gamma}\left(t_{i+2}\right)\right]}{\left(\hat{t}_{i+3}-\hat{t}_{i+2}\right)+\left(\hat{t}_{i+2}-\hat{t}_{i+1}\right)}\right. \\
& \quad-\frac{\left(t_{i+2}-t_{i+1}\right)^{1-\lambda}\left[\dot{\gamma}\left(t_{i+1}\right)+\frac{t_{i+2}-t_{i+1}}{2} \ddot{\gamma}\left(t_{i+1}\right)\right]}{\left(\hat{t}_{i+3}-\hat{t}_{i+2}\right)+\left(\hat{t}_{i+2}-\hat{t}_{i+1}\right)} \\
& +\frac{O\left(\left(t_{i+3}-t_{i+2}\right)^{2}\right)}{\left(\hat{t}_{i+3}-\hat{t}_{i+2}\right)+\left(\hat{t}_{i+2}-\hat{t}_{i+1}\right)}+\frac{O\left(\left(t_{i+2}-t_{i+1}\right)^{2}\right)}{\left(\hat{t}_{i+3}-\hat{t}_{i+2}\right)+\left(\hat{t}_{i+2}-\hat{t}_{i+1}\right)} \\
& -\frac{\left(t_{i+2}-t_{i+1}\right)^{1-\lambda}\left(\left[\dot{\gamma}\left(t_{i+1}\right)+\frac{t_{i+2}-t_{i+1}}{2} \ddot{\gamma}\left(t_{i+1}\right)\right]+\left[\dot{\gamma}\left(t_{i}\right)+\frac{t_{i+1}-t_{i}}{2} \ddot{\gamma}\left(t_{i}\right)\right]\right)}{\left(\hat{t}_{i+2}-\hat{t}_{i+1}\right)+\left(\hat{t}_{i+1}-\hat{t}_{i}\right)} \\
& +\frac{O\left(\left(t_{i+1}-t_{i}\right)^{2}\right)}{\left(\hat{t}_{i+2}-\hat{t}_{i+1}\right)+\left(\hat{t}_{i+1}-\hat{t}_{i}\right)}+\frac{\left.O \hat{t}_{i+2}-\hat{t}_{i+1}\right)+\left(\hat{t}_{i+1}-\hat{t}_{i}\right)}{\left.\left(\hat{t}_{i+1}\right)^{2}\right)} .
\end{aligned}
$$

Thus as for (3.24), formula (3.26) yields an upper bound for

$$
\begin{aligned}
& \left\|\check{\gamma}_{i}\left[\hat{t}_{i}, \hat{t}_{i+1}, \hat{t}_{i+2}, \hat{t}_{i+3}\right]\right\| \leq \frac{\left(t_{i+3}-t_{i+2}\right)^{1-3 \lambda}\left\|\dot{\gamma}\left(t_{i+2}\right)+\frac{t_{i+3}-t_{i+2}}{2} \ddot{\gamma}\left(t_{i+2}\right)\right\|}{1+O\left(\left(t_{i+3}-t_{i+2}\right)^{2}\right)} \\
& \quad+2 \frac{\left(t_{i+2}-t_{i+1}\right)^{1-3 \lambda}\left\|\dot{\gamma}\left(t_{i+1}\right)+\frac{t_{i+2}-t_{i+1}}{2} \ddot{\gamma}\left(t_{i+1}\right)\right\|}{1+O\left(\left(t_{i+2}-t_{i+1}\right)^{2}\right)} \\
& \quad+\frac{\left\|O\left(\left(t_{i+3}-t_{i+2}\right)^{2-2 \lambda}\right)\right\|}{1+O\left(\left(t_{i+3}-t_{i+2}\right)^{2}\right)}+\frac{\left\|O\left(\left(t_{i+2}-t_{i+1}\right)^{2-2 \lambda}\right)\right\|}{1+O\left(\left(t_{i+2}-t_{i+1}\right)^{2}\right)} .
\end{aligned}
$$

Hence as for proving (3.25), given $1-3 \lambda<2-2 \lambda$ (for $\lambda \in[0,1]$ ) we obtain:

$$
\check{\gamma}_{i}\left[\hat{t}_{i}, \hat{t}_{i+1}, \hat{t}_{i+2}, \hat{t}_{i+3}\right]=O\left(\delta_{m}^{1-3 \lambda}\right) .
$$

Consequently by (3.17) the following holds:

$$
\check{\gamma}_{i}^{\prime \prime \prime}(\hat{t})=O\left(\delta_{m}^{1-3 \lambda}\right) .
$$

Furthermore, again by (3.17) and (3.25) (for $\left.\hat{t} \in \psi_{i}\left(\left[t_{i}, t_{i+3}\right]\right)\right)$ we have:

$$
\check{\gamma}_{i}^{\prime \prime}(\hat{t})=O\left(\delta_{m}^{1-2 \lambda}\right)+2\left[\left(\hat{t}-\hat{t}_{i+2}\right)+\left(\hat{t}-\hat{t}_{i+1}\right)+\left(\hat{t}-\hat{t}_{i}\right)\right] \cdot O\left(\delta_{m}^{1-3 \lambda}\right) .
$$


Thus by (3.13) and Lagrange Theorem for arbitrary $\hat{t}=\psi_{i}(t) \in \psi_{i}\left(\left[t_{i}, t_{i+3}\right]\right)$ (and for $j=0,1,2$ and $t \in\left[t_{i}, t_{i+3}\right]$ ) one arrives at:

$$
\hat{t}-\hat{t}_{i+j}=\frac{\psi_{i}(t)-\psi_{i}\left(t_{i+j}\right)}{t-t_{i+j}}\left(t-t_{i+j}\right)=\dot{\psi}_{i}(\xi)\left(t-t_{i+j}\right)=O\left(\delta_{m}^{\lambda}\right) .
$$

Hence, coupling (3.28) with (3.29) results in:

$$
\check{\gamma}_{i}^{\prime \prime}(\hat{t})=O\left(\delta_{m}^{1-2 \lambda}\right) .
$$

A special case of uniform samplings $\mathcal{T}_{u}=\left\{\frac{i T}{m}\right\}_{i=0}^{m}$ (with $\beta=1$ in (1.2)) leads to some simplifications. Indeed upon incorporating into (3.23) both $t_{i+k+2}-$ $t_{i+k+1}=t_{i+k+1}-t_{i+k}=\delta_{m}$ and $\dot{\gamma}\left(t_{i+k+1}\right)=\dot{\gamma}\left(t_{i+k}\right)+O\left(\left(t_{i+k+1}-t_{i+k}\right)\right)$ the term $\delta_{m}$ factorizes in the first two expressions accelerating the third divided differences (here $0 \leq 2-2 \lambda \leq 2-\lambda$ for $\lambda \geq 0$ and $k=0,1$ ) by order one to:

$$
\check{\gamma}_{i}\left[\hat{t}_{i+k}, \hat{t}_{i+k+1}, \hat{t}_{i+k+2}\right]=O\left(\delta_{m}^{2-2 \lambda}\right)+O\left(\delta_{m}^{2-\lambda}\right)=O\left(\delta_{m}^{2-2 \lambda}\right) .
$$

A similar reduction follows in (3.26) upon using $\hat{t}_{i+3}-\hat{t}_{i+2}=\hat{t}_{i+2}-\hat{t}_{i+1}=$ $\hat{t}_{i+1}-\hat{t}_{i}$ with $\dot{\gamma}\left(t_{i+k+1}\right)=\dot{\gamma}\left(t_{i+k}\right)+O\left(t_{i+k+1}-t_{i+k}\right)$ (where $k=0,1$ ) rendering:

$$
\check{\gamma}_{i}^{\prime \prime \prime}(\hat{t})=6 \check{\gamma}_{i}\left[\hat{t}_{i}, \hat{t}_{i+1}, \hat{t}_{i+2}, \hat{t}_{i+3}\right]=O\left(\delta_{m}^{2-3 \lambda}\right)+O\left(\delta_{m}^{2-2 \lambda}\right)=O\left(\delta_{m}^{2-3 \lambda}\right) .
$$

Consequently the latter combined with (3.17), (3.29) and (3.31) yields:

$$
\check{\gamma}_{i}^{\prime \prime}(\hat{t})=O\left(\delta_{m}^{2-2 \lambda}\right)+O\left(\delta_{m}^{2-3 \lambda}\right) \cdot O\left(\delta_{m}^{\lambda}\right)=O\left(\delta_{m}^{2-2 \lambda}\right) .
$$

\subsection{Final estimation of differences $f_{i}=\check{\gamma}_{i} \circ \psi_{i}-\gamma$}

We examine now the underlying asymptotics in $f_{i}=\check{\gamma}_{i} \circ \psi_{i}-\gamma$ uniformly over each $J_{i}$ and for arbitrary $\lambda \in[0,1)$ (see (3.4)). Indeed, upon feeding (3.13), (3.14), (3.27) and (3.30) into (3.4) one obtains:

$$
\begin{aligned}
f_{i}(t)= & O\left(\delta_{m}^{4}\right) \cdot\left[O\left(\delta_{m}^{1-3 \lambda}\right) \cdot O\left(\delta_{m}^{2 \lambda-2}\right) \cdot O\left(\delta_{m}^{\lambda-2}\right)+O\left(\delta_{m}^{1-2 \lambda}\right) \cdot O\left(\delta_{m}^{2 \lambda-4}\right)\right. \\
& \left.+O\left(\delta_{m}^{1-2 \lambda}\right) \cdot O\left(\delta_{m}^{\lambda-1}\right) \cdot O\left(\delta_{m}^{\lambda-3}\right)+O(1)\right],
\end{aligned}
$$

which ultimately leads to:

$$
f_{i}(t)=O\left(\delta_{m}^{4}\right) \cdot\left[O\left(\delta_{m}^{-3}\right)+O\left(\delta_{m}^{-3}\right)+O\left(\delta_{m}^{-3}\right)+O(1)\right]=O\left(\delta_{m}^{1}\right) .
$$

The latter justifies the first claim (2.1) of Theorem 2 .

The special case of uniform samplings addressed by (2.2) follows once (3.16), (3.32) and (3.33) are incorporated into (3.4) resulting in (uniformly over $J_{i}$ ):

$$
\begin{aligned}
f_{i}(t)= & O\left(\delta^{4}\right) \cdot\left[O\left(\delta_{m}^{2-3 \lambda}\right) \cdot O\left(\delta_{m}^{2 \lambda-2}\right) \cdot O\left(\delta_{m}^{\lambda}\right)+O\left(\delta_{m}^{2-2 \lambda}\right) \cdot O\left(\delta_{m}^{2 \lambda}\right)\right. \\
& \left.+O\left(\delta_{m}^{2-2 \lambda}\right) \cdot O\left(\delta_{m}^{\lambda-1}\right) \cdot O\left(\delta_{m}^{\lambda-1}\right)+O(1)\right],
\end{aligned}
$$

which in turn yields:

$$
f_{i}(t)=O\left(\delta_{m}^{4}\right) \cdot\left[O(1)+O\left(\delta_{m}^{2}\right)+O(1)+O(1)\right]=O\left(\delta_{m}^{4}\right) .
$$

The proof is thus completed. 


\section{Sufficient conditions on reparameterization}

In this section several sufficient conditions for the cubic $\psi_{i}$ to yield a genuine reparameterization are formulated. The case of $\lambda=1$ renders $\psi_{i}$ as a parameterization for arbitrary admissible sampling (1.1) as proved in [17]. The importance of $\dot{\psi}_{i}>0$ is outlined in Section 2 - see item e). Noticeably to enforce $\psi_{i}$ as a reparameterization it suffices to let the quadratic $\dot{\psi}_{i}(t)=a_{i} t^{2}+b_{i} t+c_{i}>0$ over each $J_{i}$. The latter follows if e.g. (see Figure 1):

$$
\begin{array}{lll}
a_{i}<0 \wedge \dot{\psi}_{i}\left(t_{i}\right)>0 & \wedge \dot{\psi}_{i}\left(t_{i+3}\right)>0, \\
a_{i}>0 \wedge \dot{\psi}_{i}\left(t_{i}\right)>0 & \wedge-b_{i} / 2 a_{i}<t_{i}, \\
a_{i}>0 \wedge-b_{i} / 2 a_{i}>t_{i+3} & \wedge \dot{\psi}_{i}\left(t_{i+3}\right)>0, \\
a_{i}>0 \wedge \dot{\psi}_{i}\left(-b_{i} / 2 a_{i}\right)>0 . &
\end{array}
$$

Clearly for a given collection of $Q_{m}$ and $\mathcal{T}$, the testing of $\dot{\psi}_{i}>0$ over different $J_{i}$ can vary between constraints $(i)-(i v)$ (or between any other ones). Assuming that we admit the subfamily of more-or-less uniform samplings (1.2) satisfying $0<\beta_{0} \leq \beta \leq 1$ (with some $\beta_{0}$ fixed) the conditions (4.1) can be expressed in terms of $\beta_{0}$. A full treatment of solving (4.1) for arbitrary $\lambda \in[0,1)$ exceeds the scope and page limit for this paper. Some hints can be found in [10], where the parameterization issue for piecewise-quadratic Lagrange interpolation $\hat{\gamma}_{2}$ based on $Q_{m}$ and exponential parameterization (1.3) is thoroughly studied.

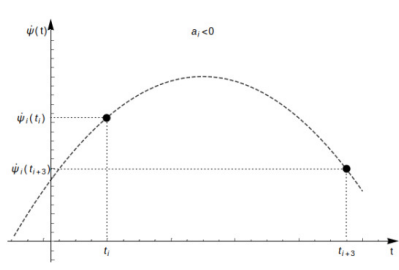

a)

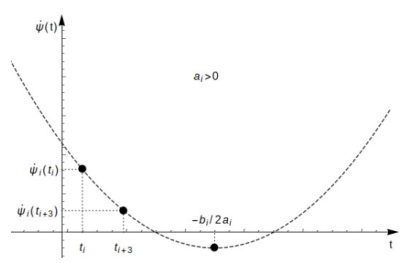

c)

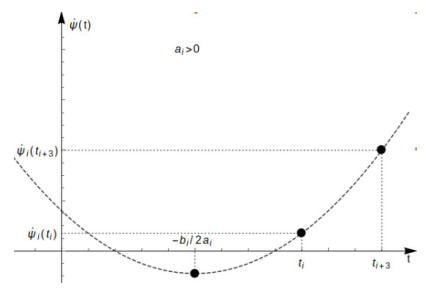

b)

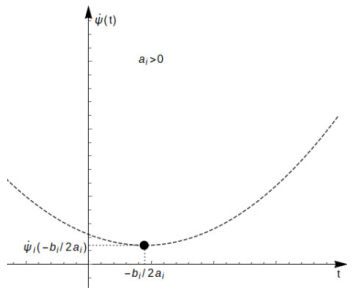

d)

Figure 1. The plot of (4.1) with $\dot{\psi}_{i}>0$ over $\left[t_{i}, t_{i+3}\right]$ for: a) (i), b) (ii), c) (iii) \& d) (iv).

\section{Sharpness of the asymptotics in trajectory estimation}

The sharpness of the asymptotics from Theorem 2 (see also Definition 4 ) is proved below. In doing so, assume the straight line $\gamma_{l}(t)=(t, 0) \in \mathbb{E}^{2}$ to be 
sampled more-or-less uniformly (over consecutive $J_{i}$ ) according to:

$$
t_{i}=\frac{i}{m}, \quad t_{i}=\frac{i+1}{m}, \quad t_{i+2}=\frac{i+2}{m} \quad \text { and } \quad t_{i+3}=\frac{i+4}{m} .
$$

Here $\delta_{m}=\frac{2}{m}$ and $\beta=\frac{1}{2}$ - see Definition 2. The reduced data $q_{i}=\gamma_{l}\left(t_{i}\right)$ are supplemented with $\hat{\mathcal{T}} \approx \mathcal{T}$ determined by the exponential parameterization (1.3). The analysis to follow (for $\lambda \in[0,1)$ ) results in

$$
f_{i}^{l}=\check{\gamma} \circ \psi_{i}-\gamma_{l}=K \delta_{m}+O\left(\delta_{m}^{\eta}\right),
$$

holding asymptotically for some $\eta>1$ (uniformly over each $J_{i}$ ). To prove the sharpness of Theorem 2 it suffices to show the latter for some particular $\bar{t} \in J_{i}$ and a special $i$. The constant $K$ and those from $O\left(\delta_{m}^{\eta}\right)$ are independent of $m$. The sharpness of (2.1) is proved in the next example.

Example 1. Given $\bar{\delta}=\delta_{m} / 2$ the sampling (5.1) over $J_{0}$ reads as:

$$
t_{0}=0, \quad t_{1}=\bar{\delta}, \quad t_{2}=2 \bar{\delta} \quad \text { and } \quad t_{3}=4 \bar{\delta} .
$$

The exponential parameterization (1.3) for $\left\{\gamma_{l}\left(t_{i}\right)\right\}_{i=0}^{3}$ yields:

$$
\hat{t}_{0}=0, \quad \hat{t}_{1}=\bar{\delta}^{\lambda}, \quad \hat{t}_{2}=2 \bar{\delta}^{\lambda} \quad \text { and } \quad \hat{t}_{3}=\left(2+2^{\lambda}\right) \bar{\delta}^{\lambda} .
$$

The corresponding divided differences for a cubic $\psi_{0}: J_{0} \rightarrow \mathbb{R}$ are equal to:

$$
\psi_{0}[0]=0, \quad \psi_{0}[0, \bar{\delta}]=\psi_{0}[\bar{\delta}, 2 \bar{\delta}]=\bar{\delta}^{\lambda-1} \quad \text { and } \quad \psi_{0}[0, \bar{\delta}, 2 \bar{\delta}]=0 .
$$

Coupling now $\psi_{0}[\bar{\delta}, 2 \bar{\delta}, 4 \bar{\delta}]=(1 / 3)\left(2^{\lambda-1}-1\right) \bar{\delta}^{\lambda-2}$ with (5.4) leads to:

$$
\psi_{0}[0, \bar{\delta}, 2 \bar{\delta}, 4 \bar{\delta}]=\frac{1}{12}\left(2^{\lambda-1}-1\right) \bar{\delta}^{\lambda-3} .
$$

Thus by (3.5), (5.2), (5.4) and (5.5) we arrive at:

$$
\psi_{0}(t)=\bar{\delta}^{\lambda-1} t+\frac{1}{12}\left(2^{\lambda-1}-1\right) \bar{\delta}^{\lambda-3} t(t-\bar{\delta})(t-2 \bar{\delta}),
$$

for any $t \in[0,4 \bar{\delta}]=J_{0}$. Substituting $\bar{t}=3 \bar{\delta} \in J_{0}$ into (5.6) yields:

$$
\psi_{0}(3 \bar{\delta})=\left(\frac{5}{2}+2^{\lambda-2}\right) \bar{\delta}^{\lambda}
$$

Similarly, by (5.3) for $\gamma_{l}$ we obtain the following:

$$
\check{\gamma}_{0}(0)=\overrightarrow{0}, \quad \check{\gamma}_{0}\left(\bar{\delta}^{\lambda}\right)=(\bar{\delta}, 0), \quad \check{\gamma}_{0}\left(2 \bar{\delta}^{\lambda}\right)=(2 \bar{\delta}, 0), \quad \check{\gamma}_{0}\left(\left(2^{\lambda}+2\right) \bar{\delta}^{\lambda}\right)=(4 \bar{\delta}, 0) .
$$

Consequently:

$$
\check{\gamma}_{0}[0]=\overrightarrow{0}, \quad \check{\gamma}_{0}\left[0, \bar{\delta}^{\lambda}\right]=\check{\gamma}_{0}\left[\bar{\delta}^{\lambda}, 2 \bar{\delta}^{\lambda}\right]=\left(\bar{\delta}^{1-\lambda}, 0\right), \quad \check{\gamma}_{0}\left[0, \bar{\delta}^{\lambda}, 2 \bar{\delta}^{\lambda}\right]=\overrightarrow{0} .
$$

In addition, as $\check{\gamma}_{0}\left[2 \bar{\delta}^{\lambda},\left(2+2^{\lambda}\right) \bar{\delta}^{\lambda}\right]=\left(2^{1-\lambda} \bar{\delta}^{1-\lambda}, 0\right)$ we have:

$$
\check{\gamma}_{0}\left[\bar{\delta}^{\lambda}, 2 \bar{\delta}^{\lambda},\left(2+2^{\lambda}\right) \bar{\delta}^{\lambda}\right]=\left(\frac{2^{1-\lambda}-1}{2^{\lambda}+1} \bar{\delta}^{1-2 \lambda}, 0\right) .
$$


Hence upon combining now (5.8) with (5.9) we arrive at:

$$
\check{\gamma}_{0}\left[0, \bar{\delta}^{\lambda}, 2 \bar{\delta}^{\lambda},\left(2+2^{\lambda}\right) \bar{\delta}^{\lambda}\right]=\left(\frac{2^{1-\lambda}-1}{\left(2^{\lambda}+1\right)\left(2+2^{\lambda}\right)} \bar{\delta}^{1-3 \lambda}, 0\right) .
$$

Again coupling together (5.3), (5.8) and (5.10) yields, for $\hat{t}=\psi_{0}(t)$ with $t \in$ $[0,4 \bar{\delta}]$, the following formula:

$$
\check{\gamma}_{0}(\hat{t})=\left(\bar{\delta}^{1-\lambda} \hat{t}+\frac{2^{1-\lambda}-1}{\left(2^{\lambda}+1\right)\left(2+2^{\lambda}\right)} \bar{\delta}^{1-3 \lambda} \hat{t}\left(\hat{t}-\bar{\delta}^{\lambda}\right)\left(\hat{t}-2 \bar{\delta}^{\lambda}\right), 0\right) .
$$

By (5.7) and (5.11) the "error difference":

$$
f_{0}^{l}(3 \bar{\delta})=\check{\gamma}_{0}\left(\psi_{0}(3 \bar{\delta})\right)-\gamma(3 \bar{\delta})=\left((1 / 2) \rho(\lambda) \delta_{m}, 0\right),
$$

where for $\lambda \in[0,1)$ we have

$$
\rho(\lambda)=\left(\frac{5}{2}+2^{\lambda-2}\right)\left(1+\left(\frac{1}{2}+2^{\lambda-2}\right)\left(\frac{3}{2}+2^{\lambda-2}\right) \frac{2^{1-\lambda}-1}{\left(2^{\lambda}+1\right)\left(2+2^{\lambda}\right)}\right)-3 .
$$

The plots of the function $\rho$ over $[0,1)$ together with its magnification over $[0.899,1)$ are presented in Figure 2. Evidently the coefficient $\rho(\lambda)$ standing along $\delta_{m}$ is non-vanishing. Noticeably $\rho(1)=0$ and thus $f_{0}^{l}(3 \bar{\delta})=0$.

A moment of reflection reveals that here both $\gamma_{l}$ and $\check{\gamma}$ coincide (since $t_{i}=\hat{t}_{i}$ ) resulting in $f^{l} \equiv 0$ over $[0, T]$. By (5.12) a linear convergence order for $f_{0}^{l}$ holds at $\hat{t}=3 \bar{\delta}$ which implies that $f^{l}$ defined as a track-sum of $f_{i}^{l}$ cannot be of faster order than a linear one (uniformly over $[0, T]$ ). On the other by $(2.1)$ the difference $f^{l}$ satisfies $O\left(\delta_{m}^{1}\right)$. Consequently the sharpness of $(2.1)$ is proved (see also Definition 4). Note that the sharpness of (2.2) for $\lambda=1$ and general admissible samplings (1.1) follows as a spin-off of calculations from Example 3.

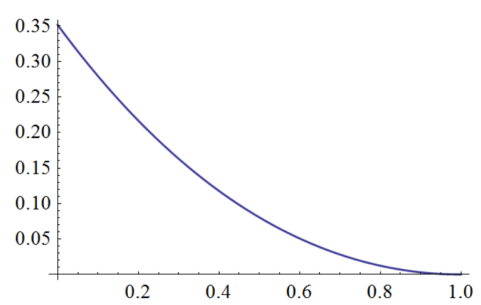

a)

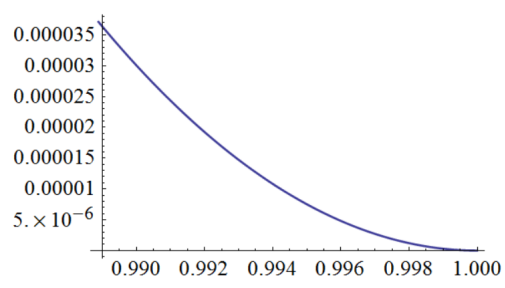

b)

Figure 2. The plot of $\rho(\lambda)>0$ from (5.12) over a) $[0,1)$ and b) $[0,899,1)$.

The next example justifies the sharpness of (2.2) (see also Definition 4) established for uniform sampling (here $\delta_{m}=T / m$ ). Without loss one may assume that $[0, T]$ is shifted left by fixed $\varepsilon>0$ to $[-\varepsilon, T-\varepsilon]$ so that, asymptotically it contains all interpolation knots $t_{i}=-2 \delta_{m}, t_{i+1}=-\delta_{m}, t_{i+2}=0$ and $t_{i+3}=\delta_{m}$. In order to simplify the notation used for $t_{i+j}$ (with $j=0,1,2,3$ ) 
we set $i=0$. The regular curve tested now coincides with $\gamma_{q}(t)=\left(t^{4}+t, 0\right)$. The asymptotics of the respective difference $h^{q}=\check{\gamma} \circ \psi-\gamma_{q}$ is examined over $J_{0}=\left[-2 \delta_{m}, \delta_{m}\right]$. The detailed symbolic calculations used in Example 2 is stored in Mathematica Notebook under the URL link [20].

Example 2. The uniform knots (with $\delta_{m}=T / m$ ):

$$
t_{0}=-2 \delta_{m}, \quad t_{1}=-\delta_{m}, \quad t_{2}=0, \quad t_{3}=\delta_{m}
$$

together with the exponential parameterization (1.3) and $\left\{\gamma_{q}\left(t_{i}\right)\right\}_{i=0}^{3}$ give (asymptotically):

$$
\begin{aligned}
& \hat{t}_{0}=0, \quad \hat{t}_{1}=\left(\delta_{m}-15 \delta_{m}^{4}\right)^{\lambda}, \quad \hat{t}_{2}=\left[\left(\delta_{m}-15 \delta_{m}^{4}\right)^{\lambda}+\left(\delta_{m}-\delta_{m}^{4}\right)^{\lambda}\right] \\
& \hat{t}_{3}=\left[\left(\delta_{m}-15 \delta_{m}^{4}\right)^{\lambda}+\left(\delta_{m}-\delta_{m}^{4}\right)^{\lambda}+\left(\delta_{m}+\delta_{m}^{4}\right)^{\lambda}\right]
\end{aligned}
$$

The divided differences of order one or two for a cubic $\psi:\left[-2 \delta_{m}, \delta_{m}\right] \rightarrow \mathbb{R}$ amount to:

$$
\begin{aligned}
& \psi\left[-2 \delta_{m}\right]=0, \quad \psi\left[-2 \delta_{m},-\delta_{m}\right]=\left(\delta_{m}-15 \delta_{m}^{4}\right)^{\lambda} / \delta_{m}, \\
& \psi\left[-\delta_{m}, 0\right]=\left(\delta_{m}-\delta_{m}^{4}\right)^{\lambda} / \delta_{m}, \quad \psi\left[0, \delta_{m}\right]=\left(\delta_{m}+\delta_{m}^{4}\right)^{\lambda} / \delta_{m}
\end{aligned}
$$

Note that we omit here the subscript 0 in $\psi_{0}$. The latter yields:

$$
\begin{aligned}
\psi\left[-2 \delta_{m},-\delta_{m}, 0\right] & =\left(\left(\delta_{m}-\delta_{m}^{4}\right)^{\lambda}-\left(\delta_{m}-15 \delta_{m}^{4}\right)^{\lambda}\right) /\left(2 \delta_{m}^{2}\right), \\
\psi\left[-\delta_{m}, 0, \delta_{m}\right] & =\left(\left(\delta_{m}+\delta_{m}^{4}\right)^{\lambda}-\left(\delta_{m}-\delta_{m}^{4}\right)^{\lambda}\right) /\left(2 \delta_{m}^{2}\right),
\end{aligned}
$$

which in turn renders:

$$
\psi\left[-2 \delta_{m},-\delta_{m}, 0, \delta_{m}\right]=\frac{\left(\delta_{m}-15 \delta_{m}^{4}\right)^{\lambda}-2\left(\delta_{m}-\delta_{m}^{4}\right)^{\lambda}+\left(\delta_{m}+\delta_{m}^{4}\right)^{\lambda}}{6 \delta_{m}^{3}} .
$$

Combining (3.5) with (5.13), (5.15), (5.16), (5.17) and upon using Collect Mathematica function (Collect $[$ Collect $[\psi[t, \lambda], \lambda], t]$ ) leads to:

$$
\begin{aligned}
& \psi(t)=\left(\delta_{m}-15 \delta_{m}^{4}\right)^{\lambda}+\left(\delta_{m}-\delta_{m}^{4}\right)^{\lambda}+\left[\left(-\left(\delta_{m}-15 \delta_{m}^{4}\right)^{\lambda}+5\left(\delta_{m}-\delta_{m}^{4}\right)^{\lambda}\right.\right. \\
& \left.\left.\quad+2\left(\delta_{m}+\delta_{m}^{4}\right)^{\lambda}\right) /\left(6 \delta_{m}\right)\right] t+\left[\left(\left(\delta_{m}+\delta_{m}^{4}\right)^{\lambda}-\left(\delta_{m}-\delta_{m}^{4}\right)^{\lambda}\right) /\left(2 \delta_{m}^{2}\right)\right] t^{2} \\
& +\left[\left(\left(\delta_{m}-15 \delta_{m}^{4}\right)^{\lambda}-2\left(\delta_{m}-\delta_{m}^{4}\right)^{\lambda}+\left(\delta_{m}+\delta_{m}^{4}\right)^{\lambda}\right) /\left(6 \delta_{m}^{3}\right)\right] t^{3}
\end{aligned}
$$

for an arbitrary $t \in\left[-2 \delta_{m}, \delta_{m}\right]$. The evaluation of $(5.18)$ at $\bar{t}=\left(\delta_{m} / 2\right)$ with Mathematica symbolic computation Collect $\left[\psi\left[\delta_{m} / 2, \lambda\right], \lambda\right]$ results in:

$$
\psi(\bar{t})=(15 / 16)\left(\delta_{m}-15 \delta_{m}^{4}\right)^{\lambda}+(5 / 4)\left(\delta_{m}-\delta_{m}^{4}\right)^{\lambda}+(5 / 16)\left(\delta_{m}+\delta_{m}^{4}\right)^{\lambda} .
$$

As $\gamma_{q}$ and $\check{\gamma}$ coincide at interpolation points $\left\{q_{i+j}\right\}_{j=0}^{3}$ we have $\check{\gamma}\left(\hat{t}_{2}\right)=\overrightarrow{0}$ and:

$$
\check{\gamma}\left(\hat{t}_{0}\right)=\left(-2 \delta_{m}+16 \delta_{m}^{4}, 0\right), \quad \check{\gamma}\left(\hat{t}_{1}\right)=\left(-\delta_{m}+\delta_{m}^{4}, 0\right), \quad \check{\gamma}\left(\hat{t}_{3}\right)=\left(\delta_{m}+\delta_{m}^{4}, 0\right) .
$$

Hence and by (5.14) the respective divided differences for $\check{\gamma}$ are equal:

$$
\begin{array}{rlrl}
\check{\gamma}\left[\hat{t}_{0}\right] & =\left(-2 \delta_{m}+16 \delta_{m}^{4}, 0\right), & \check{\gamma}\left[\hat{t}_{0}, \hat{t}_{1}\right]=\left(\left(\delta_{m}-15 \delta_{m}^{4}\right)^{1-\lambda}, 0\right), \\
\check{\gamma}\left[\hat{t}_{1}, \hat{t}_{2}\right]=\left(\left(\delta_{m}-\delta_{m}^{4}\right)^{1-\lambda}, 0\right), & \check{\gamma}\left[\hat{t}_{2}, \hat{t}_{3}\right]=\left(\left(\delta_{m}+\delta_{m}^{4}\right)^{1-\lambda}, 0\right) .
\end{array}
$$


Thus the third divided differences satisfy:

$$
\begin{aligned}
& \check{\gamma}\left[\hat{t}_{0}, \hat{t}_{1}, \hat{t}_{2}\right]=\left(\frac{-\left(\delta_{m}-15 \delta_{m}^{4}\right)^{1-\lambda}+\left(\delta_{m}-\delta_{m}^{4}\right)^{1-\lambda}}{\left(\delta_{m}-15 \delta_{m}^{4}\right)^{\lambda}+\left(\delta_{m}-\delta_{m}^{4}\right)^{\lambda}}, 0\right), \\
& \check{\gamma}\left[\hat{t}_{1}, \hat{t}_{2}, \hat{t}_{3}\right]=\left(\frac{-\left(\delta_{m}-\delta_{m}^{4}\right)^{1-\lambda}+\left(\delta_{m}+\delta_{m}^{4}\right)^{1-\lambda}}{\left(\delta_{m}-\delta_{m}^{4}\right)^{\lambda}+\left(\delta_{m}+\delta_{m}^{4}\right)^{\lambda}}, 0\right),
\end{aligned}
$$

which in turn with the aid of Mathematica Simplify function leads to:

$$
\check{\gamma}\left[\hat{t}_{0}, \hat{t}_{1}, \hat{t}_{2}, \hat{t}_{3}\right]=\left(\frac{\frac{\left(\delta_{m}-15 \delta_{m}^{4}\right)^{1-\lambda}-\left(\delta_{m}-\delta_{m}^{4}\right)^{1-\lambda}}{\left(\delta_{m}-15 \delta_{m}^{4}\right)^{\lambda}+\left(\delta_{m}-\delta_{m}^{4}\right)^{\lambda}}+\frac{-\left(\delta_{m}-\delta_{m}^{4}\right)^{1-\lambda}+\left(\delta_{m}+\delta_{m}^{4}\right)^{1-\lambda}}{\left(\delta_{m}-\delta_{m}^{4}\right)^{\lambda}+\left(\delta_{m}+\delta_{m}^{4}\right)^{\lambda}}}{\left(\delta_{m}-15 \delta_{m}^{4}\right)^{\lambda}+\left(\delta_{m}-\delta_{m}^{4}\right)^{\lambda}+\left(\delta_{m}+\delta_{m}^{4}\right)^{\lambda}}, 0\right) \text {. }
$$

Applying (5.14), (5.19), (5.20), (5.21) and (5.22) with Newton's interpolation formula yields for $h^{q}(\bar{t})=\left(\check{\gamma} \circ \psi-\gamma_{q}\right)(\bar{t})$ (with $\left.\overline{\hat{t}}=\psi(\bar{t})\right)$ the following:

$$
\begin{aligned}
& h^{q}(\bar{t})=\left(-2 \delta_{m}+16 \delta_{m}^{4}+\overline{\hat{t}}\left(\delta_{m}-15 \delta_{m}^{4}\right)^{1-\lambda}\right. \\
& \quad+\frac{\overline{\hat{t}}\left(\overline{\hat{t}}-\left(\delta_{m}-15 \delta_{m}^{4}\right)^{\lambda}\right)\left(\left(\delta_{m}-\delta_{m}^{4}\right)^{1-\lambda}-\left(\delta_{m}-15 \delta_{m}^{4}\right)^{1-\lambda}\right)}{\left(\delta_{m}-15 \delta_{m}^{4}\right)^{\lambda}+\left(\delta_{m}-\delta_{m}^{4}\right)^{\lambda}} \\
& \quad+\frac{\overline{\hat{t}}\left(\overline{\hat{t}}-\left(\delta_{m}-15 \delta_{m}^{4}\right)^{\lambda}\right)\left(\overline{\hat{t}}-\left(\delta_{m}-15 \delta_{m}^{4}\right)^{\lambda}-\left(\delta_{m}-\delta_{m}^{4}\right)^{\lambda}\right)}{\left(\delta_{m}-15 \delta_{m}^{4}\right)^{\lambda}+\left(\delta_{m}-\delta_{m}^{4}\right)^{\lambda}+\left(\delta_{m}+\delta_{m}^{4}\right)^{\lambda}} \\
& \left.\quad \times\left(\frac{\left(\delta_{m}-15 \delta_{m}^{4}\right)^{1-\lambda}-\left(\delta_{m}-\delta_{m}^{4}\right)^{1-\lambda}}{\left(\delta_{m}-15 \delta_{m}^{4}\right)^{\lambda}+\left(\delta_{m}-\delta_{m}^{4}\right)^{\lambda}}+\frac{\left(\delta_{m}+\delta_{m}^{4}\right)^{1-\lambda}-\left(\delta_{m}-\delta_{m}^{4}\right)^{1-\lambda}}{\left(\delta_{m}-\delta_{m}^{4}\right)^{\lambda}+\left(\delta_{m}+\delta_{m}^{4}\right)^{\lambda}}\right), 0\right) .
\end{aligned}
$$

Setting $\lambda=1$ or $\lambda=0$ in (5.23) and applying Mathematica Simplify function leads to $h^{q}(\bar{t})=\frac{15}{16} \delta_{m}^{4}$. The latter proves the sharpness of (2.2) for uniformly sampled regular curves and two special cases of either $\lambda=1$ or $\lambda=0$. In order to recalculate (5.23) for other $\lambda \in(0,1)$, Series $\left[f_{i}\left[\delta_{m},\left\{\delta_{m}, 0,3\right\}\right]\right.$ in Mathematica is used to expand the expressions $f_{i}$ (for $i=1,2,3$ ) into Taylor series (up to the 3rd order at 0) yielding $f_{1}\left(\delta_{m}\right)=\left(1-15 \delta_{m}^{3}\right)^{\lambda}=1-15 \lambda \delta_{m}^{3}+O\left(\delta_{m}^{4}\right), f_{2}\left(\delta_{m}\right)=$ $\left(1-\delta_{m}^{3}\right)^{\lambda}=1-\lambda \delta_{m}^{3}+O\left(\delta_{m}^{4}\right)$, and $f_{3}\left(\delta_{m}\right)=\left(1+\delta_{m}^{3}\right)^{\lambda}=1+\lambda \delta_{m}^{3}+O\left(\delta_{m}^{4}\right)$. The latter passed into (5.14) yields:

$$
\begin{aligned}
& \hat{t}_{1}=\delta_{m}^{\lambda}\left(1-15 \lambda \delta_{m}^{3}+O\left(\delta_{m}^{4}\right)\right) \\
& \hat{t}_{2}=\delta_{m}^{\lambda}\left(1-15 \lambda \delta_{m}^{3}+O\left(\delta_{m}^{4}\right)\right)+\delta_{m}^{\lambda}\left(1-\lambda \delta_{m}^{3}+O\left(\delta_{m}^{4}\right)\right) \\
& \hat{t}_{3}=\delta_{m}^{\lambda}\left(1-15 \lambda \delta_{m}^{3}+O\left(\delta_{m}^{4}\right)\right)+\delta_{m}^{\lambda}\left(1+O\left(\delta_{m}^{4}\right)\right) .
\end{aligned}
$$

Define now in Mathematica for the first coordinate of $h^{q}(t)=\check{\gamma} \circ \psi-\gamma_{q}$ the function:

$$
\text { fun }\left[\lambda_{-}\right]:=\check{\gamma}\left[\text { Factor }\left[\text { Collect }\left[\psi\left[\delta_{m} / 2, \lambda\right], \lambda\right]\right], \lambda\right]-\left(\left(\delta_{m} / 2\right)^{4}+\delta_{m} / 2\right) .
$$

Upon passing (5.24) into (5.25) (without Simplify) we obtain that

$$
\begin{aligned}
& \operatorname{fun}(\lambda)= \\
& \left(\frac{255}{16}+\frac{15}{8}(14-14 \lambda)-15 \lambda+\frac{5}{8}(-6+6 \lambda)+\frac{5}{2}(-15+15 \lambda)\right) \delta_{m}^{4}+O\left(\delta_{m}^{5}\right),
\end{aligned}
$$


or with Simplify we have $f u n(\lambda)=\frac{15}{16} \delta_{m}^{4}+O\left(\delta_{m}^{5}\right)$ - see also the Mathematica Notebook under the URL link [20]. The latter justifies the sharpness of (2.2) for the remaining $\lambda \in(0,1)$ and any uniformly sampled $\gamma \in C^{4}$.

\section{Necessity of more-or-less uniformity}

We demonstrate now that more-or-less-uniformity for (2.1) (with $\lambda \in[0,1)$ ) is essential and cannot be omitted in Theorem 2. A simple inspection shows that the admissible sampling (6.1) does not satisfy more-or-less uniformity (1.2) (with $\delta_{m} \rightarrow 0$ ). As previously we simplify the notation used for $t_{i+j}$ (with $j=0,1,2,3)$ by setting $i=0$. The asymptotics of $h^{q}(t)=(\check{\gamma} \circ \psi)(t)-$ $\gamma_{q}(t)$ is examined over the sub-interval $J_{0}=\left[-\delta_{m}, \delta_{m}\right]$. The detailed symbolic computations used in Example 3 are conducted in Mathematica Notebook stored under the URL link [21].

Example 3. Consider now the following non-more-or-less uniform sampling:

$$
t_{0}=-\delta_{m}, \quad t_{1}=0, \quad t_{2}=\delta_{m}^{2} \quad \text { and } \quad t_{3}=\delta_{m} .
$$

The exponential parameterization (1.3) applied to $\left\{\gamma_{q}\left(t_{i}\right)\right\}_{i=0}^{3}$ and (6.1) yields (asymptotically):

$$
\begin{aligned}
& \hat{t}_{0}=0, \quad \hat{t}_{1}=\left(\delta_{m}-\delta_{m}^{4}\right)^{\lambda}, \quad \hat{t}_{2}=\left(\delta_{m}-\delta_{m}^{4}\right)^{\lambda}+\left(\delta_{m}^{2}+\delta_{m}^{8}\right)^{\lambda}, \\
& \hat{t}_{3}=\left(\delta_{m}-\delta_{m}^{4}\right)^{\lambda}+\left(\delta_{m}-\delta_{m}^{2}+\delta_{m}^{4}-\delta_{m}^{8}\right)^{\lambda}+\left(\delta_{m}^{2}+\delta_{m}^{8}\right)^{\lambda} .
\end{aligned}
$$

The corresponding divided differences for a cubic $\psi: J_{0} \rightarrow \mathbb{R}$ are equal to:

$$
\begin{aligned}
& \psi\left[-\delta_{m}\right]=0, \quad \psi\left[-\delta_{m}, 0\right]=\left(\delta_{m}-\delta_{m}^{4}\right)^{\lambda} / \delta_{m}, \quad \psi\left[0, \delta_{m}^{2}\right]=\left(\delta_{m}^{2}+\delta_{m}^{8}\right)^{\lambda} / \delta_{m}^{2}, \\
& \psi\left[\delta_{m}^{2}, \delta_{m}\right]=\left(\delta_{m}-\delta_{m}^{2}+\delta_{m}^{4}-\delta_{m}^{8}\right)^{\lambda} /\left(\delta_{m}-\delta_{m}^{2}\right) .
\end{aligned}
$$

Hence latter yields:

$$
\begin{aligned}
& \psi\left[-\delta_{m}, 0, \delta_{m}^{2}\right]=-\left(\delta_{m}\left(\delta_{m}-\delta_{m}^{4}\right)^{\lambda}+\left(\delta_{m}^{2}+\delta_{m}^{8}\right)^{\lambda}\right) /\left(1+\delta_{m}\right) \delta_{m}^{3}, \\
& \psi\left[0, \delta_{m}^{2}, \delta_{m}\right]=-\frac{\delta_{m}\left(\delta_{m}-\delta_{m}^{2}+\delta_{m}^{4}-\delta_{m}^{8}\right)^{\lambda}-\left(\delta_{m}^{2}+\delta_{m}^{8}\right)^{\lambda}+\delta_{m}\left(\delta_{m}^{2}+\delta_{m}^{8}\right)^{\lambda}}{\left(-1+\delta_{m}\right) \delta_{m}^{3}},
\end{aligned}
$$

which with Mathematica Factor $\left[\psi\left[-\delta_{m}, 0, \delta_{m}^{2}, \delta_{m}\right]\right]$ results in

$$
\begin{aligned}
\psi & {\left[-\delta_{m}, 0, \delta_{m}^{2}, \delta_{m}\right]=\left(\delta_{m}\left(\delta_{m}-\delta_{m}^{4}\right)^{\lambda}+\delta_{m}^{2}\left(\delta_{m}-\delta_{m}^{4}\right)^{\lambda}\right.} \\
& -\delta_{m}\left(\delta_{m}-\delta_{m}^{2}+\delta_{m}^{4}-\delta_{m}^{8}\right)^{\lambda}+2\left(\delta_{m}^{4}+\delta_{m}^{8}\right)^{\lambda}-\delta_{m}^{2}\left(\delta_{m}-\delta_{m}^{2}+\delta_{m}^{4}-\delta_{m}^{8}\right)^{\lambda} \\
& \left.-\delta_{m}\left(\delta_{m}^{2}+\delta_{m}^{8}\right)^{\lambda}-\delta_{m}^{2}\left(\delta_{m}^{2}+\delta_{m}^{8}\right)^{\lambda}\right) /\left(2\left(-1+\delta_{m}\right)\left(1+\delta_{m}\right) \delta_{m}^{4}\right) .
\end{aligned}
$$

Combining (3.5) with (6.1), (6.3), (6.4), (6.5) with Collect $[$ Factor $[\psi[t, \lambda]], t]$ yields $\left(\right.$ where $\kappa_{1}\left(\delta_{m}\right)=\left(\delta_{m}-\delta_{m}^{2}+\delta_{m}^{4}-\delta_{m}^{8}\right)^{\lambda}$ and $\kappa_{2}\left(\delta_{m}\right)=\left(\delta_{m}^{2}+\delta_{m}^{8}\right)^{\lambda}$ over $J_{0}$ the following

$$
\begin{aligned}
& \psi(t)=\left[\left(\delta_{m}-\delta_{m}^{4}\right)^{\lambda}\left(-2 \delta_{m}^{4}+2 \delta^{6}\right)+t^{3}\left(\left(\delta_{m}-\delta_{m}^{4}\right)^{\lambda}\left(-\delta_{m}+\delta_{m}^{2}\right)\right.\right. \\
& \left.-\kappa_{1}\left(\delta_{m}\right)\left(\delta_{m}+\delta_{m}^{2}\right)+\kappa_{2}\left(\delta_{m}\right)\left(2-\delta_{m}-\delta_{m}^{2}\right)\right)+t^{2}\left(\left(\delta_{m}^{2}-\delta_{m}^{4}\right)\right. \\
& \left.\quad \times\left(\left(\delta_{m}-\delta_{m}^{4}\right)^{\lambda}-\kappa_{1}\left(\delta_{m}\right)-\kappa_{2}\left(\delta_{m}\right)\right)\right)+t\left(\left(\delta_{m}-\delta_{m}^{4}\right)^{\lambda}\left(-\delta_{m}^{4}+\delta_{m}^{5}\right)\right.
\end{aligned}
$$




$$
\left.\left.+\kappa_{1}\left(\delta_{m}\right)\left(\delta_{m}^{4}+\delta_{m}^{5}\right)+\kappa_{2}\left(\delta_{m}\right)\left(-2 \delta_{m}^{2}+\delta^{4}+\delta_{m}^{5}\right)\right)\right] /\left(2\left(-1+\delta_{m}\right)\left(1+\delta_{m}\right) \delta_{m}^{4}\right) .
$$

The evaluation of $(6.6)$ at $\bar{t}=\left(\delta_{m} / 2\right)$ with Factor $[\psi[\delta / 2, \lambda]]$ results in:

$$
\psi(\bar{t})=\frac{3\left(\left(\delta_{m}-\delta_{m}^{4}\right)^{\lambda}\left(-5 \delta_{m}-\delta_{m}^{2}+6 \delta_{m}^{3}\right)-\kappa_{1}\left(\delta_{m}\right)\left(\delta_{m}-\delta_{m}^{2}-2 \delta_{m}^{3}\right)\right)}{16\left(-1+\delta_{m}\right) \delta_{m}\left(1+\delta_{m}\right)}
$$

Since $\gamma_{q}$ and $\check{\gamma}$ coincide at interpolation points $\left\{q_{i+j}\right\}_{j=0}^{3}$ we have $\gamma\left(\hat{t}_{1}\right)=\overrightarrow{0}$,

$$
\check{\gamma}\left(\hat{t}_{0}\right)=\left(-\delta_{m}+\delta_{m}^{4}, 0\right), \quad \check{\gamma}\left(\hat{t}_{2}\right)=\left(\delta_{m}^{2}+\delta_{m}^{8}, 0\right), \quad \check{\gamma}\left(\hat{t}_{3}\right)=\left(\delta_{m}+\delta_{m}^{4}, 0\right)
$$

Hence by (6.2) the respective divided differences for $\check{\gamma}$ are equal:

$$
\begin{aligned}
& \check{\gamma}\left[\hat{t}_{0}\right]=\left(-\delta_{m}+\delta_{m}^{4}, 0\right), \quad \check{\gamma}\left[\hat{t}_{0}, \hat{t}_{1}\right]=\left(\left(\delta_{m}-\delta_{m}^{4}\right)^{1-\lambda}, 0\right) \\
& \check{\gamma}\left[\hat{t}_{1}, \hat{t}_{2}\right]=\left(\left(\delta_{m}^{2}+\delta_{m}^{8}\right)^{1-\lambda}, 0\right), \quad \check{\gamma}\left[\hat{t}_{2}, \hat{t}_{3}\right]=\left(\left(\delta_{m}-\delta_{m}^{2}+\delta_{m}^{4}-\delta_{m}^{8}\right)^{1-\lambda}, 0\right)
\end{aligned}
$$

Consequently we obtain:

$$
\begin{aligned}
& \check{\gamma}\left[\hat{t}_{0}, \hat{t}_{1}, \hat{t}_{2}\right]=\left(\frac{-\left(\delta_{m}-\delta_{m}^{4}\right)^{1-\lambda}+\left(\delta_{m}^{2}+\delta_{m}^{8}\right)^{1-\lambda}}{\left(\delta_{m}-\delta_{m}^{4}\right)^{\lambda}+\left(\delta_{m}^{2}+\delta_{m}^{8}\right)^{\lambda}}, 0\right) \\
& \check{\gamma}\left[\hat{t}_{1}, \hat{t}_{2}, \hat{t}_{3}\right]=\left(\frac{\left(\delta_{m}-\delta_{m}^{2}+\delta_{m}^{4}-\delta_{m}^{8}\right)^{1-\lambda}-\left(\delta_{m}^{2}+\delta_{m}^{8}\right)^{1-\lambda}}{\left(\delta_{m}-\delta_{m}^{2}+\delta_{m}^{4}-\delta_{m}^{8}\right)^{\lambda}+\left(\delta_{m}^{2}+\delta_{m}^{8}\right)^{\lambda}}, 0\right),
\end{aligned}
$$

which in turn leads to $\left(\lambda_{1}=1-\lambda\right)$ :

$$
\check{\gamma}\left[\hat{t}_{0}, \hat{t}_{1}, \hat{t}_{2}, \hat{t}_{3}\right]=\left(\frac{\frac{\left(\delta_{m}-\delta_{m}^{4}\right)^{\lambda_{1}}-\left(\delta_{m}^{2}+\delta_{m}^{8}\right)^{\lambda_{1}}}{\left(\delta_{m}-\delta_{m}^{4}\right)^{\lambda}+\left(\delta_{m}^{2}+\delta_{m}^{8}\right)^{\lambda}}+\frac{\left(\delta_{m}-\delta_{m}^{2}+\delta_{m}^{4}-\delta_{m}^{8}\right)^{\lambda_{1}}-\left(\delta_{m}^{2}+\delta_{m}^{8}\right)^{\lambda_{1}}}{\left(\delta_{m}-\delta_{m}^{2}+\delta_{m}^{4}-\delta_{m}^{8}\right)^{\lambda}+\left(\delta_{m}^{2}+\delta_{m}^{8}\right)^{\lambda}}}{\left(\delta_{m}-\delta_{m}^{4}\right)^{\lambda}+\left(\delta_{m}-\delta_{m}^{2}+\delta_{m}^{4}-\delta_{m}^{8}\right)^{\lambda}+\left(\delta_{m}^{2}+\delta_{m}^{8}\right)^{\lambda}}, 0\right) .
$$

Incorporating (6.2), (6.7), (6.8), (6.9) and the latter into Newton's interpolation formula yields for

$$
\begin{aligned}
& h^{q}(\bar{t})=\left(\check{\gamma} \circ \psi-\gamma_{q}\right)(\bar{t})=\left(-\frac{3 \delta_{m}}{2}+\frac{15 \delta_{m}^{4}}{16}+\left(\delta_{m}-\delta_{m}^{4}\right)^{1-\lambda} \overline{\hat{t}}\right. \\
& +\frac{\left(\left(\delta_{m}^{2}+\delta_{m}^{8}\right)^{1-\lambda}-\left(\delta_{m}-\delta_{m}^{4}\right)^{1-\lambda}\right) \overline{\hat{t}}\left(\overline{\hat{t}}-\left(\delta_{m}-\delta_{m}^{4}\right)^{\lambda}\right)}{\left(\delta_{m}-\delta_{m}^{4}\right)^{\lambda}+\left(\delta_{m}^{2}+\delta_{m}^{8}\right)^{\lambda}} \\
& \quad+\overline{\hat{t}}\left(\overline{\hat{t}}-\left(\delta_{m}-\delta_{m}^{4}\right)^{\lambda}\right)\left(\overline{\hat{t}}-\left(\delta_{m}-\delta_{m}^{4}\right)^{\lambda}-\left(\delta_{m}^{2}+\delta_{m}^{8}\right)^{\lambda}\right) \\
& \quad \times \frac{\left.\frac{\left(\delta_{m}-\delta_{m}^{4}\right)^{1-\lambda}-\left(\delta_{m}^{2}+\delta_{m}^{8}\right)^{1-\lambda}}{\left(\delta_{m}-\delta_{m}^{4}\right)^{\lambda}+\left(\delta_{m}^{2}+\delta_{m}^{8}\right)^{\lambda}}+\frac{\left(\delta_{m}-\delta_{m}^{2}+\delta_{m}^{4}-\delta_{m}^{8}\right)^{1-\lambda}-\left(\delta_{m}^{2}+\delta_{m}^{8}\right)^{1-\lambda}}{\left(\delta_{m}-\delta_{m}^{2}+\delta_{m}^{4}-\delta_{m}^{8}\right)^{\lambda}+\left(\delta_{m}^{2}+\delta_{m}^{8}\right)^{\lambda}}, 0\right),}{\left(\delta_{m}-\delta_{m}^{4}\right)^{\lambda}+\left(\delta_{m}-\delta_{m}^{2}+\delta_{m}^{4}-\delta_{m}^{8}\right)^{\lambda}+\left(\delta_{m}^{2}+\delta_{m}^{8}\right)^{\lambda}},
\end{aligned}
$$


where $\overline{\hat{t}}=\psi(\bar{t})$. Setting now e.g. $\lambda=0$ in (6.10) and using Mathematica Simplify to $h^{q}(\bar{t})$ leads to (see Mathematica Notebook under the URL link [21]):

$$
\begin{aligned}
h^{q}(\bar{t})= & \frac{-144+432 \delta_{m}+328 \delta_{m}^{2}+296 \delta_{m}^{3}-5437 \delta_{m}^{4}-2757 \delta_{m}^{5}+17663 \delta_{m}^{6}}{8192 \delta_{m}^{2}\left(-1+\delta_{m}^{2}\right)^{3}} \\
& +\frac{7605 \delta_{m}^{7}-25274 \delta_{m}^{8}-8438 \delta_{m}^{9}+8577 \delta_{m}^{10}+9801 \delta_{m}^{11}}{8192 \delta_{m}^{2}\left(-1+\delta_{m}^{2}\right)^{3}} \\
& +\frac{13845 \delta_{m}^{12}-5895 \delta_{m}^{13}-19566 \delta_{m}^{14}+1116 \delta_{m}^{15}+7560 \delta_{m}^{16}}{8192 \delta_{m}^{2}\left(-1+\delta_{m}^{2}\right)^{3}} .
\end{aligned}
$$

Visibly the latter is of the sharp order:

$$
h^{q}(\bar{t})=(-144 / 8192) \delta_{m}^{-2}+O\left(\delta_{m}^{-1}\right),
$$

not coinciding with the linear asymptotics from Theorem 2 established for any $\lambda \in[0,1)$ and arbitrary more-or-less uniform sampling. Such deceleration effect is due to the fact that sampling (6.1) does not satisfy (1.2). A similar sharp asymptotics with the slowest term $K_{\lambda} \delta_{m}^{-2}$ (with $K_{\lambda} \neq 0$ ) follows upon substituting any $\lambda \in[0,1)$ into (6.10) and applying Mathematica Simplify function. Due to the page limitation the Mathematica Notebook is attached (see ( [21])) so that the latter can be verified upon performing symbolic computation for any fixed $\lambda \in[0,1)$. Note also that feeding $\lambda=1$ into (6.10) results in:

$$
h^{q}(\bar{t})=\frac{3}{16}\left(1-2 \delta_{m}\right) \delta_{m}^{4}
$$

which yields an exact quartic convergence order in trajectory estimation at $\bar{t}=\left(\delta_{m} / 2\right)$. The latter justifies sharpness of Theorem 2 claimed also for $\lambda=1$ and the general class of admissible samplings. Evidently, though (6.1) does not fulfill more-or-less uniformity it still complies however with (1.1).

\section{Experiments}

We verify now numerically the sharpness of the asymptotics established in Theorem 2 for some regular $2 \mathrm{D}$ and $3 \mathrm{D}$ curves. All tests are carried out in Mathematica 10.0 (see [22]) and resort to either uniform or more-or-less uniform samplings (with $t_{i} \in[0,1]$ and for $i=0,1, \ldots, m$ ) defined according to:

$$
t_{i}= \begin{cases}i / m+1 /(2 m), & \text { for } i=4 k+1, \\ i / m-1 /(2 m), & \text { for } i=4 k+3, \\ i / m, & \text { for } i \text { even }\end{cases}
$$

and

$$
\text { (i) } t_{i}=i / m+(-1)^{i+1} /(3 m), \quad(i i) t_{i}=i / m .
$$

Here, $\beta=1$ for $(7.2)(i i), \beta=(1 / 3)$ for $(7.1)$ and $\beta=(1 / 5)$ for $(7.2)(i)$ - see Definition 2. For a given $m$, the error $E_{m}$, between two continuous functions $\gamma$ and $\hat{\gamma}_{3}$ reads as $E_{m}=\max _{[0,1]}\left\|\left(\hat{\gamma}_{3} \circ \psi_{3}\right)(t)-\gamma(t)\right\|$. To estimate numerically 
the convergence order $\alpha(\lambda)$ in trajectory approximation, first $\left\{E_{m}\right\}_{m=m_{\min }}^{m_{\max }}$ is calculated, where $m_{\min }$ and $m_{\max }$ are sufficiently large fixed constants. Next a linear regression yielding a function $y(x)=\bar{\alpha}(\lambda) x+b$ is applied to $\left\{\left(\log (m),-\log \left(E_{m}\right)\right)\right\}_{m=m_{\min }}^{m_{\max }}$. Mathematica built-in function LinearModelFit extracts a coefficient $\bar{\alpha}(\lambda) \approx \alpha(\lambda)$. The tests conducted here use the following three different $C^{\infty}$ regular curves: a spiral $\gamma_{s p}$ and a cubic $\gamma_{o}$ in $\mathbb{E}^{2}$ and a Steinmetz curve $\gamma_{s t}$ in $\mathbb{E}^{3}$.

Example 4. Consider a planar spiral $\gamma_{s p}:[0,1] \rightarrow \mathbb{E}^{2}$ defined by $\gamma_{s p}(t)=$ $((0.2+t) \cos (\pi(1-t)),(0.2+t) \sin (\pi(1-t)))$ and sampled according to either (7.1) or (7.2). The numerical results for $\bar{\alpha}(\lambda) \approx \alpha(\lambda)$ listed in Table 1 for different $\lambda \in[0,1]$, render the asymptotics consistent with this from Theorem 2 .

Table 1. $\bar{\alpha}(\lambda) \approx \alpha(\lambda)$ for $\gamma_{s p}$ sampled as in (7.1) and (7.2) with $m \in\{96, \ldots, 120\}$.

\begin{tabular}{cccccccc}
\hline$\lambda$ & 0.0 & 0.1 & 0.3 & 0.5 & 0.7 & 0.9 & 1.0 \\
\hline $\bar{\alpha}(\lambda)$ for $(7.1)$ & 1.005 & 1.009 & 1.021 & 1.038 & 1.076 & 1.269 & 4.006 \\
$\bar{\alpha}(\lambda)$ for $(7.2)(i)$ & 1.028 & 1.028 & 1.026 & 1.023 & 1.015 & 1.007 & 4.071 \\
\hline$\alpha(\lambda)$ in Theorem 2 & 1.0 & 1.0 & 1.0 & 1.0 & 1.0 & 1.0 & 4.0 \\
\hline $\bar{\alpha}(\lambda)$ for $(7.2)(i i)$ & 4.027 & 4.020 & 4.013 & 4.037 & 4.047 & 4.060 & 4.067 \\
\hline$\alpha(\lambda)$ in Theorem 2 & 4.0 & 4.0 & 4.0 & 4.0 & 4.0 & 4.0 & 4.0 \\
\hline
\end{tabular}

Example 5. Assume now a regular Steinmetz 3D curve $\gamma_{s t}:[0,1] \rightarrow \mathbb{E}^{3}$ defined as $\gamma_{s t}(t)=\left(\cos (2 \pi t), \sin (2 \pi t), \sqrt{1.2^{2}-(\sin (2 \pi t))^{2}}\right)$ is sampled along either (7.1) or (7.2). The computed $\bar{\alpha}(\lambda) \approx \alpha(\lambda)$ for various $\lambda \in[0,1]$ are presented in Table 2. Thus the sharpness of (2.1) and (2.2) is numerically confirmed.

Table 2. $\bar{\alpha}(\lambda) \approx \alpha(\lambda)$ for $\gamma_{s t}$ sampled as in (7.1) and (7.2) with $m \in\{72, \ldots, 162\}$.

\begin{tabular}{cccccccc}
\hline$\lambda$ & 0.0 & 0.1 & 0.3 & 0.5 & 0.7 & 0.9 & 1.0 \\
\hline $\bar{\alpha}(\lambda)$ for $(7.1)$ & 1.000 & 1.000 & 1.003 & 1.001 & 1.008 & 1.200 & $4.981^{* *}$ \\
$\bar{\alpha}(\lambda)$ for $(7.2)(i)$ & 0.993 & 0.994 & 0.996 & 0.998 & 1.009 & 1.186 & $3.961^{* *}$ \\
\hline$\alpha(\lambda)$ in Theorem 2 & 1.0 & 1.0 & 1.0 & 1.0 & 1.0 & 1.0 & 4.0 \\
\hline $\bar{\alpha}(\lambda)$ for $(7.2)(i i)^{*}$ & 3.977 & 3.972 & 3.965 & 3.950 & 3.954 & 3.950 & 3.948 \\
$\alpha(\lambda)$ in Theorem 2 & 4.0 & 4.0 & 4.0 & 4.0 & 4.0 & 4.0 & 4.0 \\
\hline
\end{tabular}

*for $159 \leq m \leq 201 \quad * *$ for $201 \leq m \leq 240$

Example 6. Finally, consider a planar regular curve $\gamma_{o}:[0,1] \rightarrow \mathbb{E}^{2}$ given by $\gamma_{o}(t)=\left(\cos ^{2}(t), 1.5^{t}\right)$ and sampled as previously according to either (7.1) or 
Table 3. $\bar{\alpha}(\lambda) \approx \alpha(\lambda)$ for $\gamma_{o}$ sampled as in (7.1) and (7.2) with $m \in\{72, \ldots, 162\}$.

\begin{tabular}{cccccccc}
\hline$\lambda$ & 0.0 & 0.1 & 0.3 & 0.5 & 0.7 & 0.9 & 1.0 \\
\hline $\bar{\alpha}(\lambda)$ for $(7.1)$ & 0.998 & 0.998 & 0.998 & 0.999 & 0.999 & 1.005 & $3.91^{* *}$ \\
$\bar{\alpha}(\lambda)$ for $(7.2)(i)$ & 0.999 & 0.999 & 0.999 & 0.999 & 1.000 & 1.010 & $3.94^{*}$ \\
\hline$\alpha(\lambda)$ in Theorem 2 & 1.0 & 1.0 & 1.0 & 1.0 & 1.0 & 1.0 & 4.0 \\
\hline $\bar{\alpha}(\lambda)$ for $(7.2)(i i)$ & 3.999 & 3.943 & 3.915 & $3.952^{*}$ & $3.947^{*}$ & $3.946^{*}$ & $3.95^{*}$ \\
\hline$\alpha(\lambda)$ in Theorem 2 & 4.0 & 4.0 & 4.0 & 4.0 & 4.0 & 4.0 & 4.0 \\
\hline
\end{tabular}

$*_{\text {for }} 159 \leq m \leq 201 \quad * *$ for $330 \leq m \leq 387$

(7.2). The respective estimates $\alpha \overline{(\lambda)}$ of $\alpha(\lambda)$ (for varying $\lambda \in[0,1]$ ) are presented in Table 3. Visibly, the asymptotics established in Theorem 2 (together with its sharpness) is as above, numerically verified in affirmative.

\section{Conclusions}

The main results and the motivation of this work are fully listed in Section 2 (see items a)-f)). The principal findings can be summarized as follows:

Section 3 proves Theorem 2 which extends Theorem 1 holding merely for $\lambda=1$. The combination of the latter yields a surprising abrupt discontinuity in convergence orders while estimating $\gamma$ with $\hat{\gamma}_{3} \circ \psi_{3}$. Here a fast quartic order $\alpha(1)=4$ drops to the linear one $\alpha(\lambda)=1$ holding for any $\lambda \in[0,1)$ incorporated into the exponential parameterization (1.3).

Section 4 formulates several sufficient conditions for $\psi_{3}:[0, T] \rightarrow[0, \hat{T}]$ to be a reparameterization (see (4.1)). Geometrical meaning of (4.1) is also given.

Section 5 justifies the sharpness of (2.1) and (2.2) with the aid of non-trivial analytic and symbolic computations in accordance with Definition 4.

Section 6 justifies the necessity of more-or-less uniformity (2) in proving Theorem 2. The analytic argument combined with symbolic computation is employed. Additionally, the case of $\lambda=1$ relies merely on a general class of admissible samplings (1.1) as also confirmed herein.

Section 7 verifies independently with the aid of numerical tests performed in Mathematica the sharpness of the asymptotics established in Theorem 2.

Section 2 specifies the main motivation standing behind this paper including desirable parameterization or non-parametrization cases of $\psi_{3}$. The related literature in the context of specific applications to fit $Q_{m}$ in conjunction with exponential parametrization is also listed.

Future work may involve $C^{2}$ cubics splines (see e.g. [1]) and (1.3) as an extension of $C^{0}$ piecewise-cubic non-parametric interpolation discussed in this paper. The case of $C^{1}$ modified Hermite interpolation is covered in [7] or [8]. 


\section{Acknowledgements}

This research was supported in part by PLGrid Infrastructure.

\section{References}

[1] C. de Boor. A practical guide to splines. Springer-Verlag, Berlin Heidelberg New York, 2001.

[2] M.P. do Carmo. Differential geometry of curves and surfaces. Prentice-Hall, Inc., Englewood Cliffs, New Jersey, 1976.

[3] M.P. Epstein. On the influence of parameterization in parametric interpolation. SIAM J. Numer. Anal., 13(2):261-268, 1976. https://doi.org/10.1137/0713025.

[4] G. Farin. Curves and surfaces for computer aided geometric design. Academic Press, San Diego, 1993.

[5] M. Janik, R. Kozera and P. Kozioł. Reduced data for curve modeling - applications in graphics, computer vision and physics. Adv. Sci. Tech., 7(18):38-35, 2013. https://doi.org/10.5604/20804075.1049599.

[6] R. Kozera. Curve modeling via interpolation based on multidimensional reduced data. Studia Informatica, 25(4B-61):1-140, 2004. Available from Internet: https://dx.doi.org/10.21936/si2004_v25.n4B.

[7] R. Kozera and M. Wilkołazka. A modified Hermite interpolation with exponential parameterization. Math. Comput. Sci., 2018. https://doi.org/10.1007/s11786018-0362-4.

[8] R. Kozera and L. Noakes. $\mathrm{C}^{1}$ interpolation with cumulative chord cubics for length and trajectory estimation. Fund. Inform., 61(3-4):281-301, 2004.

[9] R. Kozera and L. Noakes. Piecewise-quadratics and exponential parameterization for reduced data. Appl. Math. Comput., 221:620-638, 2013. https://doi.org/10.1016/j.amc.2013.06.060.

[10] R. Kozera and L. Noakes. Piecewise-quadratics and reparameterizations for interpolating reduced data. In V.P. Gerdt, W. Koepf, W.M. Seiler and E.V. Vorozhtsov(Eds.), Computer Algebra in Scientific Computing, Proc. Int. Conf. CASC 2015, volume 9301 of LNCS, pp. 260-274, Aachen, Germany, 2015. Springer International Publishing. https://doi.org/10.1007/978-3-319-240213_20.

[11] R. Kozera and L. Noakes. Exponential parameterization and $\varepsilon$-uniformly sampled reduced data. Appl. Math. Inf. Sci., 10(1):33-48, 2016. https://doi.org/10.18576/amis/100104.

[12] R. Kozera and L. Noakes. Optimal knots selection for sparse reduced data. In F. Huang and A. Sugimoto(Eds.), Image and Video Technology - Pacific Symposium on Image and Video Technology Workshops, Proc. Workshops PSIVT 2015, volume 9555 of $L N C S$, pp. 3-14, Auckland, New Zealand, 2016. Springer International Publishing, AG Switzerland. https://doi.org/10.1007/978-3-31930285-0_1.

[13] R. Kozera, L. Noakes and P. Szmielew. Quartic orders and sharpness in trajectory estimation for smooth cumulative chord cubics. In L.J. Chmielewski, R. Kozera, Bok-Suk Shin and K. Wojciechowski(Eds.), Computer Vision and Graphics, Proc. Int. Conf. ICCVG 2014, volume 8671 of LNCS, pp. 9-16, Warsaw, 
Poland, 2014. Springer International Publishing. https://doi.org/10.1007/9783-319-11331-9_2.

[14] B.I. Kvasov. Methods of shape-preserving spline approximation. World Scientific, Singapore, 2000. https://doi.org/10.1142/4172.

[15] E.T.Y. Lee. Choosing nodes in parametric curve interpolation. Comput. Aided Design, 21(6):363-370, 1989. https://doi.org/10.1016/0010-4485(89)90003-1.

[16] J. Milnor. Morse theory. Ann. of Math. Stud. 51, Princeton Univ. Press, 1963.

[17] L. Noakes and R. Kozera. Cumulative chords piecewise-quadratics and piecewisecubics. Comput. Imaging Vision, Geometric Properties from Incomplete Data, 32:59-75, 2006. https://doi.org/10.1007/1-4020-3858-8_4.

[18] A. Prokopenya, M. Minglibayev and G. Mayemerova. Symbolic calculations in studying the problem of three bodies with variable masses. Program. Comput. Softw., 40(2):79-85, 2014. https://doi.org/10.1134/S036176881402008X.

[19] A. Ralston. A first course in numerical analysis. McGraw-Hill, 1965.

[20] Mathematica Notebook 1. Available at https://goo.gl/6R6ouv.

[21] Mathematica Notebook 2. Available at https://goo.gl/vz8y8f.

[22] S. Wolfram. The Mathematica book. Wolfram Media, 5th ed., 2003. 